\title{
Accuracy and Credal Imprecision
}

\author{
DOMINIK BERGer \\ University of North Carolina at Chapel Hill \\ NiLANJAN DAS \\ University College London
}

\begin{abstract}
Many have claimed that epistemic rationality sometimes requires us to have imprecise credal states (i.e., credal states representable only by sets of credence functions) rather than precise ones (i.e., credal states representable by single credence functions). Some writers have recently argued that this claim conflicts with accuracycentered epistemology, i.e., the project of justifying epistemic norms by appealing solely to the overall accuracy of the doxastic states they recommend. But these arguments are far from decisive. In this essay, we prove some new results, which show that there is little hope for reconciling the rationality of credal imprecision with accuracy-centered epistemology.
\end{abstract}

\section{Introduction}

Let an agent have precise credences if and only if her doxastic state can be represented by a single credence function that assigns numerical values to various propositions. The value that a credence function assigns to a proposition reflects the agent's degree of belief or her confidence in that proposition. Though this way of representing doxastic states has several advantages, ${ }^{1}$ many have argued that we ought to reject it. Instead, they have proposed an imprecise model of doxastic states: on this picture, an agent's doxastic state is not represented by a single credence function, but rather by a set of credence functions called a representor by van Fraassen (1990). This view comes in two varieties. The first is the descriptive claim that, given their limited cognitive capacities, human beings like us can't possibly have precise credences. ${ }^{2}$ The second is the normative claim that there are certain scenarios where agents are permitted and required by epistemic rationality not to have precise credences. ${ }^{3}$

A standard motivation for the second claim comes from cases of non-specific evidence. Consider the following example:

(c) 2019 The Authors Nous Published by Wiley Periodicals, Inc.

This is an open access article under the terms of the Creative Commons

Attribution-NonCommercial-NoDerivs License, which permits use and distribution in any medium, provided the original work is properly cited, the use is non-commercial and no modifications or adaptations are made. 
MYSTERY COIN. You have a coin that was made at a factory where they can make coins of pretty much any bias. You have no idea whatsoever what bias your coin has. What should your credence be that when you toss the coin, it will land heads? ${ }^{4}$

How should the defender of precise credences answer this question? She could say that you should assign a credence of 0.5 to the proposition that the coin will land heads. But some find this answer to be problematic. For them, if you were rationally certain that the coin was fair, then you could indeed rationally adopt a precise credence of 0.5 . Or, if you had precise rational degrees of belief about what the possible biases of the coin are, and if the expected chance of the coin landing heads in light of those degrees of belief were 0.5 , then you could rationally assign a credence of 0.5 to the relevant proposition. But in this case, your evidence is non-specific: you have no evidence about the outcome of the coin flip other than the evidence that the chance distribution over the two outcomes of the coin flip could be anything. In the absence of any specific information whatsoever about the bias of the coin, assigning a sharp credence of 0.5 is irrational. For the defender of imprecise credences, non-specific evidence warrants non-specific credal states. So, the appropriate doxastic attitude to adopt in this example is a set of those credences that are compatible with the possible objective chances of the coin landing heads given your evidence. ${ }^{5}$ This might indeed be the entire unit interval $[0,1]$. Thus, your doxastic state should be representable by a set of credence functions which assign these values to the relevant proposition.

To fix ideas for the purposes of this paper, let an agent's total evidence with respect to a partition $X$ (where a partition is a set of mutually exclusive and exhaustive propositions) be non-specific if and only if, for some non-singleton set of probability functions $C$, the only evidence an agent has concerning the propositions in $X$ is that the objective chance function for $X$ is in $C$. Our verdict about MYSTERY COIN motivates the following principle.

Requirement of Imprecision. Suppose an agent's doxastic state is defined over a partition $X$. If the agent's total evidence is non-specific with respect to $X$, then the agent is required by epistemic rationality to adopt an imprecise credal state with respect to the propositions in $X$.

Recently, Schoenfield (2015) has given an argument (also presented in a different form by Seidenfeld, Schervish, and Kadane (2012) and Mayo-Wilson and Wheeler (2016)) that Requirement of Imprecision cannot be reconciled with accuracy-centered epistemology, i.e., the approach to epistemology on which requirements of epistemic rationality must be justified by appealing to accuracy-based considerations alone. Schoenfield shows that given certain plausible assumptions about measures of accuracy, for any imprecise credal state that is defined over a two-cell partition over the possibility space, there is a precise credal state that matches it with respect to accuracy. Since an agent cannot be required to have such an imprecise credal state from an accuracy-centered standpoint, Requirement of Imprecision is false.

However, Schoenfield's argument doesn't rule out the possibility that from an accuracy-centered standpoint, (i) in situations of non-specific evidence like MYSTERY 
COIN, an agent may always be rationally permitted to adopt imprecise credal states, and (ii) in some of those cases, at least, she may even be rationally required to adopt imprecise credal states. In particular, the following claims might still be true:

Permission for Imprecision. Suppose an agent's doxastic state is defined over a partition $X$. If the agent's total evidence is non-specific with respect to $X$, then the agent is permitted by epistemic rationality to adopt an imprecise credal state with respect to the propositions in $X$.

Weak Requirement of Imprecision. There are some bodies of non-specific evidence $E$ such that if an agent's total evidence is $E$, then, for some partition $X$, she is required by epistemic rationality to adopt an imprecise credal state with respect to the propositions in $X$.

Requirement of Imprecision, when combined with other assumptions, entails these claims. ${ }^{6}$ But these claims can also be independently motivated on the basis of cases like MYSTERY COIN. For example, in MYSTERY COIN, you cannot be required to adopt any sharp credence about the outcome of the coin flip. In particular, since all that you know is that the objective chance of heads coming up is in the set $[0,1]$, it is permissible for you to make an imprecise credal assignment of $[0,1]$ to the proposition that the coin will land heads. This consideration supports Permission for Imprecision. Moreover, under ordinary circumstances, our attitudes are defined over sets of propositions much more complex than a two-cell partition of the possibility space. If we intuitively understand MYSTERY COIN as a case of that sort, then we may indeed be correct in thinking that there is a rational requirement to adopt an imprecise credal state in this scenario. This supports Weak Requirement of Imprecision.

In this essay, we argue that the defender of imprecise credences cannot easily reconcile Weak Requirement of Imprecision and Permission for Imprecision with accuracy-centered epistemology. We show that the following two claims come out true given certain constraints on measures of accuracy: (i) for any imprecise credal state, there is a precise credal state that is just as accurate, and (ii) many imprecise credal states that are predicted to be rational by a natural precisification of Permission for Imprecision are in fact accuracy-dominated by precise credal states, i.e., guaranteed to do worse accuracy-wise than some precise credal states. If (i) is true, Weak Requirement of Imprecision cannot be accommodated within accuracycentered epistemology; if (ii) is true, there will be a tension between Permission for Imprecision and accuracy-centered epistemology. Since the constraints we impose on measures of accuracy are not easy to reject, we end up with the conclusion that there is no easy way of accommodating the rationality of imprecise credences within an accuracy-centered approach to epistemology.

Here is how we shall proceed. First, we will present Schoenfield's argument against Requirement of Imprecision $(§ 1)$. Then, we will show how her argument fails to rule out the possibility that the accuracy-centered epistemologist can accommodate Weak Requirement of Imprecision and Permission for Imprecision ( $\$ 2)$. Then, we will show that given certain plausible constraints on measures of accuracy, we 
cannot easily reconcile these two theses with accuracy-centered epistemology (\$\$34). Next, we shall consider three responses to this argument, all of which involve rejecting one or more of these constraints on measures of accuracy (\$\$5-7). We go on to argue that none of these responses can easily succeed.

\section{Accuracy and Credal Imprecision}

In this section, we introduce accuracy-centered epistemology, and present an argument given by Schoenfield (2015) that makes trouble for Requirement of Imprecision.

\subsection{Accuracy-centered epistemology}

Accuracy-centered epistemology is a species of epistemic utility theory. Epistemic utility theory has two aims. On the one hand, it seeks to articulate a conception of epistemic value that explains what makes one doxastic state more valuable from an epistemic standpoint than another. On the other hand, it seeks to explain why certain requirements of epistemic rationality have the force that they have, by showing that conforming to them is the best means towards promoting the relevant kind of epistemic value.

Accuracy-centered epistemologists think that the only source of epistemic value for credal states (i.e., doxastic states representable by credence functions or sets of credence functions) is the gradational accuracy of the credences they involve, i.e., their proximity to the truth. ${ }^{7}$ This view is sometimes called Credal Veritism. For example, the epistemic value of a doxastic state that is representable by a single credence function will depend on the gradational accuracy of the credences it assigns to various propositions, where the gradational accuracy of a credence in a true proposition is higher when the credence is closer to 1 , while the gradational accuracy of a credence in a false proposition is higher when the credence is closer to 0 . Using this conception of epistemic value, accuracy-centered epistemologists argue that various epistemic norms, such as Probabilism (the norm of having probabilistically coherent credences) and Conditionalization (the norm of adopting as posteriors one's prior conditional probabilities), can be shown to cohere with the instrumentally rational pursuit of accuracy. ${ }^{8}$ That explains why these norms have the force that they seem to have.

Accuracy-centered epistemologists assess the accuracy of precise credal states (i.e., doxastic states representable by single credence functions) using measures of accuracy. Suppose $W$ is a set of worlds, such that any set $P$ of worlds in $W$ is a proposition. Let $X=\left\{P_{1}, P_{2}, P_{3}, \ldots\right\}$ be a partition over $W$, where each cell $P_{i}$ in $X$ is a proposition. Let $B_{X}$ be the set of all credence functions defined over $X .^{9}$ The accuracy of a credence function $b$ in $B_{X}$ is measured by a precise global accuracy measure $G_{X}: B_{X} \times W \rightarrow[0,1]$, which maps a credence function $b$ in $B_{X}$ and a world $w$ in $W$ to a real value $G_{X}(b, w)$ between 0 and 1 .

There is a lot of discussion in the literature about which measures of accuracy are best suited to assess the accuracy of an agent's credal state. Instead of engaging in any such discussion, we will take the following three properties of global accuracy 
measures for granted. Relative to any partition $X$ over a set of worlds $W$, if $G_{X}$ is a legitimate global accuracy measure for credence functions defined over $X$, then

(1) Upper Bound. For any $w$ in $W$ and any proposition $P$ in $X$, if $w$ is in $P$, then $G_{X}$ will assign the maximal accuracy score of 1 to any credence function $b$ that assigns 1 to $P$ and 0 to every other proposition in $X$.

(2) Lower Bound. For any $w$ in $W$ and any proposition $P$ in $X$, if $w$ is in $P$, then $G_{X}$ will assign the minimal accuracy score of 0 to any credence function $b$ that assigns 0 to $P$ and 1 to every other proposition in $X$.

(3) Continuity. $G_{X}$ will be continuous through the space of probability functions, in the sense that, for any $w$ in $W$, small differences in credence functions defined over $X$ should result in small differences in the accuracy score of those credence functions in $w$.

\subsection{Measuring the accuracy of imprecise credal states}

This framework is only suitable for measuring the accuracy of precise credal states. How do we, then, measure the accuracy of imprecise credal states, i.e., doxastic states that aren't representable by single credence functions? The answer is simple: we introduce global accuracy measures that take as input not individual credence functions, but rather sets of credence functions. In other words, we define a new imprecise global accuracy measure $G_{X} *: \wp\left(B_{X}\right) \times W \rightarrow[0,1]$ (where $\wp\left(B_{X}\right)$ is the power set of $B_{X}$ ) which takes a set of credence functions $C$ and a world $w$ in $W$ as input and outputs an accuracy score $G_{X} *(C, w)$.

Schoenfield (2015) proposes three constraints that such imprecise accuracy measures should satisfy, and we add a fourth to make her argument work within our framework.

The first constraint is:

Extension. For any set of worlds $W$ and a partition $X$ over $W$, if $G_{X} *$ is a legitimate imprecise global accuracy measure for sets of credence functions defined over $X$, then there exists a legitimate global accuracy measure $G_{X}$ for credence functions such that $G_{X} *$ is an extension of $G_{X}$.

In other words, for any $b$ in $B_{X}$, the accuracy score that $G_{X} *$ assigns to the singleton set $\{b\}$ in a world $w$ is the same as the accuracy score that $G_{X}$ assigns to $b$ in $w$ : i.e., $G_{X} *(\{b\}, w)=G_{X}(b, w)$.

Extension is motivated by the idea that if a method of measuring the accuracy of imprecise credal states is to be legitimate, it should be consistent with at least one legitimate way of measuring the accuracy of precise credal states.

The second constraint is:

Boundedness. For any set of worlds $W$ and a partition $X$ over $W$, if $G_{X} *$ is a legitimate imprecise global accuracy measure for sets of credence functions defined over $X$, then $G_{X}{ }^{*}$ always assigns a real value between 0 and 1 (inclusive).

In particular, for any $w$ in $W$ and any $\mathrm{b}$ in $B_{X}$, 
(i) $G_{X} *(\{b\}, w)=1$ iff for any $P$ in $X$, (i) if $w$ is in $P$, then $b(P)=1$ and (ii) if $w$ is not in $P$, then $b(P)=0$.

(ii) $G_{X} *(\{b\}, w)=0$ iff for any $P$ in $X$, (i) if $w$ is in $P$, then $b(P)=0$ and (ii) if $w$ is not in $P$, then $b(P)=1$.

Boundedness is motivated by two ideas. The first is the idea that, just like legitimate global accuracy measures for precise credences, legitimate imprecise accuracy measures must also output real values; otherwise, given Extension, it will be difficult to compare the accuracy of imprecise credal states with precise ones. The second is the idea that, even for imprecise credal states, there seems to be a maximally accurate credal state and a minimally accurate credal state for every possible world. The maximally accurate one is the one that assigns credence 1 to all those propositions that are true in that world and 0 to all those propositions that are false in that world, while the minimally accurate one is the one that assigns credence 1 to all those propositions that are false in that world and 0 to all those propositions that are true in that world.

The third constraint is:

Probabilistic Admissibility. For any set of worlds $W$ and a partition $X$ over $W$, if $G_{X}{ }^{*}$ is a legitimate imprecise global accuracy measure for sets of credence functions defined over $X$, then for any set $C$ of probability functions in $B_{X}$, there is no set $C^{*}$ of credence functions in $B_{X}$ such that $C$ is weakly accuracy-dominated by $C^{*}$ according to $G_{X}$ *.

In other words, there is no set $C^{*}$ in $B_{X}$ such that

(i) for every world $w$ in $W, G_{X} *(C, w) \leq G_{X} *\left(C^{*}, w\right)$, and

(ii) there exists a world $w$ in $W$ such that $G_{X} *(C, w)<G_{X} *\left(C^{*}, w\right){ }^{10}$

Probabilistic Admissibility captures the thought that a legitimate global measure of accuracy shouldn't allow a probabilistic credal state to be weakly accuracydominated by other credal states. One reason for this is that, intuitively, for every probabilistic credal state, an agent could have some body of evidence that would make it rationally permissible for her to have that credal state. For example, for any set of probability functions $C$ defined over a partition $X$, she could always get evidence that the objective chance distribution over $X$ lies in that set $C$. Then, provided she has no further information about the propositions in $X$, it would be rationally permissible for the agent to adopt $C$ as her imprecise credal state. But, if $C$ is weakly accuracy-dominated by some other set $C^{*}$, then, from an accuracycentered standpoint, it would be rationally impermissible for an agent to adopt it. ${ }^{11}$

The final constraint (which we need to prove Schoenfield's result within our framework) is:

World-Invariance. For any set of worlds $W$ and a partition $X$ over $W$, if $G_{X}{ }^{*}$ is a legitimate imprecise global accuracy measure for sets of credence functions defined over $X$, then for any two worlds $w$ and $w^{*}$, if $w$ and $w^{*}$ are in the same cell of the partition $X$, then, for any set $C$ of credence functions in $B_{X}$, $G_{X}(C, w)=G_{X}\left(C, w^{*}\right) .^{12}$ 
World-Invariance says that if two worlds $w$ and $w^{*}$ assign the same truth-values to the same propositions in a partition, then a credal state defined over that partition should have the same accuracy score in the two worlds. In other words, the accuracy of a credal state depends completely on the truth-values of the propositions that it is defined over: the accuracy score of the same credal state shouldn't differ from one world to another unless the worlds assign different truth-values to the propositions over which the credal state is defined.

\subsection{Schoenfield's result}

Schoenfield (2015) begins with a principle about epistemic rationality that seems to fall out of accuracy-centered epistemology. Credal Veritists are committed to the thesis that gradational accuracy is the only source of epistemic value for credal states. Most of them also assume that an agent can be required by epistemic rationality to adopt a credal state $D$ rather than a credal state $D^{*}$ only if $D$ gives the agent a better shot at having accurate credences than $D^{*}$. Thus, they are committed to the following principle:

The Permission Principle. For any credal state $D$, if, according to every legitimate global accuracy measure, there exists a credal state $D^{*}$ that is no less accurate than $D$ in every (epistemically) possible world, then there can be no rational requirement to adopt $D .{ }^{13}$

Schoenfield shows that if we accept the Permission Principle, we must give up Requirement of Imprecision.

In particular, she proves the following claim.

Proposition 1. For any set of worlds $\mathrm{W}$ and a two-cell partition $\mathrm{X}$ over $\mathrm{W}$, let $\mathrm{C}$ be a set of probability functions in $\mathrm{B}_{\mathrm{X}}$ (the set of credence functions defined over X). If Extension, Boundedness, Probabilistic Admissibility, and World-Invariance are true, then, for any legitimate imprecise global accuracy measure $\mathrm{G}_{\mathrm{X}}{ }^{*}$, there will be a probability function $\mathrm{b}$ in $\mathrm{B}_{\mathrm{X}}$ such that, for any $\mathrm{w}$ in $\mathrm{W}, \mathrm{G}_{\mathrm{X}} *(\mathrm{C}, \mathrm{w})=\mathrm{G}_{\mathrm{X}} *(\{\mathrm{~b}\}, \mathrm{w}) .{ }^{14}$

If we grant that Extension, Boundedness, Probabilistic Admissibility, and WorldInvariance are correct, then Schoenfield has shown us that, for any imprecise credal state defined over a two-cell partition of the possibility space, there is a precise credal state which matches it with respect to accuracy.

To illustrate the main idea behind the proof, consider the case of MYSTERY COIN. In that example, there are just two propositions we care about: the proposition $H$ (i.e., the set of worlds where the coin lands heads) and the proposition $T$ (i.e., the set of worlds where the coin lands tails). So $\{H, T\}$ is the two-cell partition (over a set of worlds $W$ ) over which the agent's credal state is defined. Now, the defender of Requirement of Imprecision may be committed to thinking that in MYSTERY COIN, an agent who has no evidence about the bias of the coin is required to have an imprecise probabilistic credal state over $\{H, T\}$. Let this imprecise probabilistic credal state be represented by a set of probability functions $C$. Schoenfield then shows that there is a precise probability function $b$ that is just as accurate as $C$ 
in every world. Her argument goes like this. Let $G_{X} *$ be any legitimate imprecise global accuracy measure and let $G_{X} *\left(\mathrm{C}, w_{h}\right)=r$ for any $w_{h}$ in $H$. By Boundedness, Extension, and Continuity, we can show that there is a singleton set of a probability function $\{b\}$ such that $G_{X} *\left(\{b\}, w_{h}\right)=r$ for all $w_{h}$ in $H$. So, $\{b\}$ is just as accurate as $C$ in every $H$-world according to $G_{X} *$. But then $\{b\}$ will also have to be as accurate as $C$ in every $T$-world $w_{t}$ according to $G_{X}{ }^{*}$. Otherwise, either $\{b\}$ or $C$ will (weakly) accuracy-dominate the other and thereby violate Probabilistic Admissibility. But, then, since $\{H, T\}$ is a partition, $\{b\}$ is just as accurate as $C$ in every world according to $G_{X}$ *.

What Proposition 1 shows is that provided we accept the relevant constraints on global accuracy measures for imprecise credences, for any imprecise probabilistic credal state defined over $\{H, T\}$, there is a precise probabilistic credal state which is just as accurate as the imprecise one. So, by the Permission Principle, the agent cannot rationally be required to adopt the imprecise doxastic state in that scenario. Hence, Requirement of Imprecision is false.

Proposition 1 might also lead to a tension between Requirement of Imprecision and accuracy-centered epistemology in another way. Many authors accept the following constraint on global accuracy measures.

Strict Immodesty. If a rational agent assesses the epistemic value of various credal states in light of her own credal state according to a legitimate global accuracy measure, she should come to regard her own credal state as uniquely optimal, or as giving her the uniquely best shot at having accurate doxastic states.

The main idea behind Strict Immodesty is that a rational agent takes her doxastic state to be better than all others from an epistemic standpoint. When we are concerned solely about precise credal states, Strict Immodesty is sometimes spelled out as the requirement of strict propriety on precise global accuracy measures: any legitimate precise global accuracy measure $G_{X}$ must be such that, for any probability function $p$ defined over $X$, the expected accuracy of $p$ according to $p$ itself should be greater than the expected accuracy of any other credence function $b$ according to $p .^{15}$

Proposition 1 demonstrates that no legitimate accuracy measure for imprecise credences can satisfy Extension, Boundedness, Probabilistic Admissibility, WorldInvariance, and Strict Immodesty: if Extension, Boundedness, Probabilistic Admissibility, World-Invariance are correct, then an agent who has an imprecise credal state defined over a two-cell partition will find another precise doxastic state to be just as accurate as her own at every world. According to Strict Immodesty, such an agent cannot be rational. If we want to accommodate Strict Immodesty (as well as these other constraints on measures of accuracy) and assume that epistemic rationality does not lay down conflicting requirements, we should reject the idea that an agent can be required (or even permitted) by epistemic rationality to have imprecise doxastic states defined over two-cell partitions over the possibility space. Thus, Requirement of Imprecision must be false. Seidenfeld, Schervish, and Kadane (2012) 
and Mayo-Wilson and Wheeler (2016) prove similar results with slightly different assumptions for agents who only have doxastic attitudes towards one proposition.

\section{Responses}

In conjunction with the Permission Principle or Strict Immodesty, Schoenfield's result entails that, given certain plausible constraints on measures of accuracy, an accuracy-centered epistemologist cannot accept Requirement of Imprecision. In this section, we show that Schoenfield's result fails to rule out Weak Requirement of Imprecision and Permission for Imprecision.

\subsection{Weak Requirement of Imprecision}

It is hard to miss a certain feature of Schoenfield's result: it deals with a scenario where an agent's doxastic state only assigns credences to two propositions. This makes it difficult to see how it bears on versions of MYSTERY COIN where an agent has doxastic attitudes not only towards the two possible outcomes of the coin flip, but also towards other propositions. Consider a version of the scenario where the agent additionally has doxastic attitudes towards propositions about the chances of the two outcomes. In fact, one might think that this indeed is the more accurate description of the case: it is because the agent cannot really form any precise opinion about the bias of the coin that she cannot form a precise degree of belief about the outcomes of the coin flip. Therefore, her doxastic attitudes toward the chancepropositions are relevant to her doxastic attitudes regarding the outcomes of the coin flip.

Now, it is by no means obvious that Schoenfield's proof can be generalized to such examples. The proof crucially relies on the premise that for two sets of probability functions $C$ and $C^{*}$, if, for any heads-world $w_{h}, C$ and $C^{*}$ are equally accurate in $w_{h}$, then, for any tails-world $w_{t}, C$ and $C^{*}$ also have to be equally accurate in $w_{t}$ (or else either $\mathrm{C}$ or $C^{*}$ would be weakly accuracy-dominated). In other words, for any heads-world $w_{h}$ and any tails-world $w_{t}$, if $G_{X} *\left(C, w_{h}\right)=G_{X} *\left(C^{*}, w_{h}\right)$, then $G_{X} *\left(C, w_{t}\right)=G_{X} *\left(C^{*}, w_{t}\right)$. But in a scenario where an agent also has beliefs about the bias of the coin, let the partition be $\{F H, F T, B H, B T\}$, where $F H$ and $F T$ together include all and only those worlds where the coin is fair, while $B H$ and $B T$ include all and only those worlds where it is biased towards heads or tails. Now, the mere fact that, for any $F H$-world $w_{f h}, G_{X} *\left(C, w_{f h}\right)=G_{X} *\left(C^{*}, w_{f h}\right)$, implies nothing about how $C$ and $C^{*}$ should score in worlds where $F H$ isn't true. It is, for example, perfectly plausible that, for some $F T$-world $w_{f t}, G_{X} *\left(C, w_{f t}\right)>G_{X} *\left(C^{*}, w_{f t}\right)$, as long as $C$ does worse than $C^{*}$ in some of the worlds where $B H$ or $B T$ are true. Therefore, Schoenfield's argument does not by itself show that there cannot be any accuracyrelated reasons to adopt an imprecise doxastic state in cases where our doxastic states are defined over a more finely grained partition over the set of possible worlds (which we take it happens all the time).

Hence, the defender of imprecise credences may bite the bullet on the case that Schoenfield's proof deals with, and say that in cases where an agent's doxastic state is defined over a two-cell partition, the agent isn't rationally required to 
adopt imprecise credences. However, she might insist that there are other cases where an agent whose credal state is defined over a more complex partition is required by epistemic rationality to adopt imprecise credences. Hence, even if the Permission Principle is correct, Weak Requirement of Imprecision can still be true with respect to imprecise credal states defined over partitions with more than two cells. This is significant: since, ordinarily, our doxastic states are defined over such complex partitions, we may think that, when saddled with non-specific evidence of the relevant kind, we are often required by epistemic rationality to have imprecise credences.

\subsection{Permission for Imprecision}

Now, one might point out that even though Schoenfield's argument (when combined with the Permission Principle) doesn't rule out Weak Requirement of Imprecision, it does create trouble for Permission for Imprecision when combined with Strict Immodesty. For the argument predicts that in a case like MYSTERY COIN where the agent has non-specific evidence and adopts a credal state defined over a two-cell partition, if the credal state in question is imprecise, the agent will violate the requirement laid down by Strict Immodesty. But if Permission for Imprecision is correct, then it is indeed rationally permissible for the agent to adopt an imprecise credal state under such circumstances. So, there is a conflict between what Permission for Imprecision allows and what Strict Immodesty requires. Hence, if we accept Strict Immodesty, we would have to say that Permission for Imprecision is false. But intuitively, it seems that, given how limited her information about the bias of the coin is, the agent wouldn't be violating any requirement of rationality if she were to adopt an imprecise credal state in that scenario!

However, the argument from Strict Immodesty isn't persuasive. As Mayo-Wilson and Wheeler (2016) note, the traditional justification for Strict Immodesty, due to de Finetti (1937), is that accuracy measures that satisfy Strict Immodesty are pragmatically valuable to the inquirer: if a rational agent estimates the value of her doxastic state according to a strictly immodest measure of accuracy, she will always prefer to report her actual doxastic state rather than some other state (because she expects it to lead to the best results). This pragmatic justification may not be convincing to someone who wants to know why from a purely epistemic standpoint a rational agent should use strictly immodest measures of accuracy. The other philosophical motivation that Joyce (2009) mentions for Strict Immodesty is that violations of this constraint give rise to Moore-paradoxical situations in which an agent adopts a certain doxastic state but thinks some other doxastic state would be better from an epistemic standpoint. But this only motivates a weaker requirement:

\footnotetext{
Mild Immodesty. If a rational agent assesses the epistemic value of various credal states in light of her own credal state according to a legitimate global accuracy measure, she shouldn't come to regard her own credal state as suboptimal, or as giving her a worse shot at having accurate doxastic states, in comparison with other doxastic states.
} 
Now, note that Mild Immodesty, when combined with Proposition 1 and the other constraints on global measures of accuracy, doesn't predict that it is rationally impermissible for the agent to adopt an imprecise credal state with respect to a two-cell partition. In a case where an agent adopts an imprecise credal state with respect to a two-cell partition, if the agent measures the accuracy of doxastic states according to a mildly immodest measure of accuracy, she will think that her imprecise credal state is just as good as another precise credal state, but won't think it is worse than the precise credal state. So, it may indeed be rationally permissible to have that imprecise credal state. Thus, Permission for Imprecision can still be true. This, in turn, may help us preserve the intuition that in a case like MYSTERY COIN where our evidence is non-specific, we aren't doing anything rationally impermissible in adopting imprecise credences.

In this section, we have shown that even though Schoenfield's argument rules out Requirement of Imprecision, it leaves open the possibility that an accuracycentered epistemologist can accept Weak Requirement of Imprecision and Permission for Imprecision. In the next part of this essay, we offer a new argument to show that given certain plausible constraints on measures of accuracy, Weak Requirement of Imprecision and Permission for Imprecision cannot be reconciled with accuracycentered epistemology.

\section{Local Accuracy Measures}

Before we offer our argument, we need some additional assumptions. In particular we think that it is important to pay special attention to the distinction between local measures of accuracy (the measures of accuracy for credal assignments that an agent makes to individual propositions) and global measures of accuracy (i.e., the measures of accuracy for entire credal states), and the idea that there has to be some connection between the two.

\subsection{Measuring the local accuracy of precise credal assignments}

Recall our earlier definition of global accuracy measures for precise credal states. Given a partition $X$ over a set of worlds $W$, a global accuracy measure $G_{X}$ takes as input any credence function $b$ defined over $X$ and a world $w$, and outputs the accuracy score of $b$ in w. Standardly, the global accuracy of a credence function $b$ in a world $w$ is thought to depend on the accuracy score of the credence that it assigns to each proposition $P$ in the partition $X$. To measure the accuracy score of each credal assignment to a proposition, we need local measures of accuracy.

In order to define local measures of accuracy, we need another notion. For any world $\mathrm{w}$ in a set of worlds $W$, let an alethic vindication function $v_{w}: \wp(W) \rightarrow\{0,1\}$ be a function from a proposition $P$ to 0 and 1 such that, for any proposition $P$, $v_{w}(P)=1$ iff $w$ is in $P$ and $v_{w}(P)=0$ iff $\mathrm{w}$ is not in $P$. Intuitively, the alethic vindication function outputs the truth-value of a proposition at a particular world. If the world belongs to a proposition, then the proposition is true, and therefore the truth-value is represented by 1 . If the world doesn't belong to a proposition, the proposition is false, and therefore the truth-value is represented by 0 . 
Now, we can say what local measures of accuracy are. A precise local measure of accuracy $L:[0,1] \times\{0,1\} \rightarrow[0,1]$ takes as input (i) any credence $b(P)$ assigned to a proposition $P$ and (ii) the truth-value of $P$ at a world $w$, given by $v_{w}(P)$. It then outputs a real value $L\left(b(P), v_{w}(P)\right)$ between 0 and 1 . If we evaluate $b(P)$ at a world $w$ where $P$ is true, the local accuracy of $b(P)$ in that state is given by $L(b(P), I)$ (since $w$ is in $P$ ). If we evaluate $b(P)$ at some other world $w^{*}$ where $P$ is false, the local accuracy of $b(p)$ in that state will be given by $L(b(p), 0)$ (since $w$ is not in $P$ ).

We shall assume that precise local measures of accuracy are subject to the following constraints. If $L$ is a legitimate local measure of accuracy, then

(1) Upper Bound. If a proposition $P$ is true (/false), then a credal assignment of $1(/ 0)$ to $P$ should have the maximal accuracy score of 1 . In other words, $L(1,1)=L(0,0)=1$.

(2) Lower Bound. If a proposition $P$ is true (/false), then a credal assignment of $0(/ 1)$ to $P$ should have the minimal accuracy score of 0 . In other words, $L(0,1)=L(1,0)=0$.

(3) Continuity. The functions $L(., 0)$ and $L(., 1)$ are continuous through the unit interval $[0,1]$, in the sense that small differences in the inputs to these functions should result in small differences in their outputs.

(4) Truth-Directedness. For any precise credal assignments $r_{1}$ and $r_{2}$ in $[0,1]$,

(i) If $\left|1-r_{1}\right|<\left|1-r_{2}\right|$, then $L\left(r_{1}, 1\right)>L\left(r_{2}, 1\right)$, and

(ii) If $\left|0-r_{1}\right|<\left|0-r_{2}\right|$, then $L\left(r_{1}, 0\right)>L\left(r_{2}, 0\right) .{ }^{16}$

Here, Upper Bound, Lower Bound, and Continuity are similar to the constraints we imposed on legitimate global measures of accuracy. Truth-Directedness captures the natural thought that, from an accuracy-centered standpoint, if one credence $r_{l}$ is closer to the actual truth-value of a proposition than another credence $r_{2}$, then $r_{1}$ should have a better accuracy score than $r_{2}$.

\subsection{Measuring the local accuracy of imprecise credal assignments}

Next, we define local accuracy measures for imprecise credal assignments. An imprecise local accuracy measure $L^{*}: \wp([0,1]) \times\{0,1\} \rightarrow[0,1]$ is a function that maps a set of credences assigned to a proposition and the truth-value of that proposition to a number between 0 and 1 . Suppose we are dealing with an imprecise credal state defined over a partition $X$ over the set of worlds $W$. Suppose this credal state is represented by a set of credence functions $C$. Then, for any proposition $P$ in $X$, let the set of credences that this credal state assigns to $P$ be $C(P)=\{b(P): b \in C\}$. So, the local accuracy of $C(P)$ in a world $w$ will be $L^{*}\left(C(P), v_{w}(P)\right)$.

We impose the following constraints on imprecise local accuracy measures.

Local Extension. If $L^{*}$ is a legitimate imprecise local accuracy measure, then there exists a legitimate precise local accuracy measure $L$ such that $L^{*}$ is an extension of $L$. 
In other words, for any real number $r$ between 0 and 1 and any truth-value $v$, $L^{*}(\{r\}, v)=L(r, v)$.

Like Extension, Local Extension is motivated by the idea that local accuracy measures for imprecise credal assignments should be consistent with the manner in which we measure the accuracy of precise credal assignments.

The second constraint is:

Local Boundedness. If $L^{*}$ is a legitimate imprecise local accuracy measure, then it only assigns real values between 0 and 1 (inclusive).

In particular, for any set of credences $R$,

(i) $L^{*}(R, 0)=1$ iff $R=\{0\}$ and $L^{*}(R, 1)=1$ iff $R=\{1\}$.

(ii) $L^{*}(R, 0)=0$ iff $R=\{1\}$ and $L^{*}(R, 1)=0$ iff $R=\{0\}$.

Like Boundedness, Local Boundedness is motivated by two ideas. The first is the idea that, just like legitimate local accuracy measures for precise credences, legitimate local imprecise accuracy measures must also output real values; otherwise, given Local Extension, it will be difficult to compare the accuracy of imprecise credal assignments with precise ones. The second idea is that, even for imprecise credal assignments, there seems to be a maximally accurate credal assignment and a minimally accurate credal assignment for every proposition given its actual truth-value. When the proposition is true, the credal assignment that has maximal accuracy is the one that assigns a precise credence of 1 (here represented as $\{1\}$ ) and the credal assignment that has minimal accuracy is the one that assigns a precise credence of 0 (here represented as $\{0\}$ ). When the proposition is false, the credal assignment that has maximal accuracy is the one that assigns a precise credence of 0 and the credal assignment that has minimal accuracy is the one that assigns a precise credence of 1 .

The third constraint is:

Local Admissibility. If $L^{*}$ is a legitimate imprecise local accuracy measure, then for two sets of credences $R$ and $R^{*}, R^{*}$ doesn't strongly accuracy-dominate $R$, i.e., it is not the case that $L^{*}(R, 0)<L^{*}\left(R^{*}, 0\right)$ and $L^{*}(R, 1)<L^{*}\left(R^{*}, 1\right)$.

Just as Probabilistic Admissibility rules out the possibility that one probabilistic credal state can weakly accuracy-dominate another, Local Admissibility rules out the possibility that one credal assignment might strongly accuracy-dominate another. ${ }^{17}$ The motivating intuition here is (roughly) that violations of Local Admissibility will lead to violations of Probabilistic Admissibility simpliciter. Consider a set of probability functions $C$ defined over a partition $\mathrm{X}$, which contains a proposition $P$ such that $C(P)=R$. Suppose, contrary to Local Admissibility, some $R^{*}$ strongly accuracy-dominates $R$ given some local measure of accuracy $L^{*}$. Then we can construct another set of credence functions $C^{*}$ such that, for any proposition $P^{*}$ in $X$ other than $P, C^{*}\left(P^{*}\right)=C\left(P^{*}\right)$, but $C^{*}(P)=R^{*}$. In any world, for any proposition $P^{*}$ other than $P$, the local accuracy of the credal assignments made by $C^{*}$ will be the same as the local accuracy of the credal assignments made by $C$, but the local 
accuracy of the credal assignment made by $C^{*}$ to $P$ will always be greater than that made by $C$ to $P$. But then, intuitively, it seems that the global accuracy of $C^{*}$ must be greater than that of $C$ in every world. ${ }^{18}$ So, $C^{*}$ will accuracy-dominate $C$. Since $C$ is a set of probability functions, this will lead to a violation of Probabilistic Admissibility. Thus, in order to avoid violations of Probabilistic Admissibility we need to rule out the possibility that some credal assignments $R$ can be locally accuracy dominated.

The fourth, and most crucial, constraint, is:

Local-Global Connection. Suppose $X$ is a partition over a set of worlds $W$. Then,

(i) For any legitimate imprecise global measure of accuracy $G_{X}{ }^{*}$, there exists a legitimate imprecise local measure of accuracy $L^{*}$ such that for any two sets $C$ and $C^{*}$ of credence functions defined over $X$, and any world $w$ in $W$, if $L^{*}\left(C(P), v_{w}(P)\right)=L^{*}\left(C^{*}(P), v_{w}(P)\right)$ for every proposition $P$ in $X$, then $G_{X} *(C, w)=G_{X} *\left(C^{*}, w\right)$.

(ii) For any legitimate imprecise local measure of accuracy $L^{*}$, there exists a legitimate imprecise global measure of accuracy $G_{X}{ }^{*}$, such that for any two sets $C$ and $C^{*}$ of credence functions defined over $\mathrm{X}$, and any world $w$ in $W$, if $L^{*}\left(C(P), v_{w}(P)\right)=L^{*}\left(C^{*}(P), v_{w}(P)\right)$ for every proposition $P$ in $X$, then $G_{X} *(C, w)=G_{X} *\left(C^{*}, w\right)$.

Most accuracy-centered epistemologists also accept a version of Local-Global Connection restricted to precise credences. ${ }^{19}$ Local-Global Connection arises out of the important thought that the global accuracy score of an entire credal state should depend solely on the accuracy scores of the particular credal assignments that it makes to various propositions. We want to leave it open precisely how the global accuracy score arises from the local ones, and only impose two weak constraints by means of Local-Global Connection. Constraint (i) says that for any legitimate global accuracy measure, there exists a legitimate local accuracy measure such that if two credal states make equally accurate credal assignments to each proposition in a partition according to the local measure, then the global accuracy of the two states is the same according to the global measure. By contrast, (ii) says that for any legitimate local accuracy measure, there exists a legitimate global accuracy measure, such that if two credal states make equally accurate credal assignments to each proposition in a partition according to the local measure, then the global accuracy of the two states is the same according to the global measure.

Local Extension, Local Boundedness, Local Admissibility, and Local-Global Connection are the four constraints on measures of accuracy for imprecise credences that we need to generalize Schoenfield's argument.

\section{The Two Results}

In §2, we saw that Schoenfield's argument against Requirement of Imprecision is not decisive: it leaves open the possibility that some weaker principles such as Permission for Imprecision or Weak Requirement of Imprecision might still be true. We now show 
that if we pay close attention to the connection between local and global accuracy measures, these weaker principles won't succeed either. We prove two results in this section. The first is directed towards Weak Requirement of Imprecision and generalizes Schoenfield's proof to partitions of any number of cells. The second is directed towards Permission for Imprecision, which shows that some imprecise credal states, which are predicted to be rational by a natural precisification of Permission for Imprecision, will always be accuracy-dominated.

\subsection{The first result}

We can now show that the following claim is true:

Proposition 2. Suppose an imprecise credal state defined over a partition $\mathrm{X}$ over a set of worlds $\mathrm{W}$ is represented by a set $\mathrm{C}$ of functions in $\mathrm{B}_{\mathrm{X}}$. If Local Extension, Local Boundedness, Local Admissibility, and Local-Global Connection are true, then there will be a credence function $\mathrm{b}$ in $\mathrm{B}_{\mathrm{X}}$ such that for any legitimate imprecise global measure of accuracy $\mathrm{G}_{\mathrm{X}} * \mathrm{G}_{\mathrm{X}} *(\mathrm{C}, \mathrm{w})=\mathrm{G}_{\mathrm{X}} *(\{\mathrm{~b}\}, \mathrm{w})$ in every world $\mathrm{w}$.

Proposition 2 shows that, according to certain plausible measures of accuracy, for any imprecise credal state, there will be a precise credal state which is just as accurate as that state. This result is simply a generalization of Schoenfield's argument. Schoenfield shows that for any imprecise probabilistic credal state defined over a two-cell partition, there is a precise probabilistic credal state that is just as accurate. We have shown that, given our constraints, this isn't just true for probabilistic imprecise credal states defined over two-cell partitions, but for all imprecise credal states defined over partitions with any number of cells.

If we accept the Permission Principle, Local Extension, Local Boundedness, LocalGlobal Connection, and Local Admissibility, we will end up with the result that there are no circumstances under which an agent is rationally required to have an imprecise credal state. Hence, this shows that if the Permission Principle is true, then Weak Requirement of Imprecision cannot be true: there is no body of evidence $E$ such that an agent is rationally required to adopt an imprecise credal state in response to $E$.

However, one might think that this result still leaves open the possibility that there are scenarios like MYSTERY COIN, where the non-specificity of the agent's evidence at least makes it rationally permissible for her to adopt imprecise credences. In other words, this result remains compatible with Permission for Imprecision.

\subsection{The second result}

We will now show that, given our constraints on measures of accuracy, Permission for Imprecision is problematic as well. According to Permission for Imprecision, if an agent's total evidence is non-specific with respect to a partition $X$, then the agent is permitted by epistemic rationality to adopt an imprecise credal state. Recall that an agent's total evidence with respect to a partition $\mathrm{X}$ is non-specific if and only if, for some non-singleton set of probability functions $C$, the only evidence an agent has concerning the propositions in $\mathrm{X}$ is that the objective chance function for $X$ is in $C$. In a case where an agent's total evidence is non-specific in this sense, what 
imprecise credal state is the agent rationally permitted to adopt? The most natural answer is that it's just the credal state represented by the set of probability functions $C$. This yields:

Permission for Imprecision Precisified. For any non-singleton set of probability functions $C$, if the only evidence an agent has concerning the propositions in $X$ is that the objective chance function for $X$ is in $C$, then the agent is permitted by epistemic rationality to adopt an imprecise credal state represented by $C$.

In what follows, we show that some imprecise credal states, which are predicted to be rationally permissible by Permission for Imprecision Precisified, will always be accuracy-dominated for any plausible way of measuring the accuracy of imprecise credal states.

To see why, we need to introduce two more principles. The first is a principle that most accuracy-centered epistemologists accept.

The Non-Dominance Principle. From an epistemic standpoint, it is rationally impermissible for an agent to adopt a credal state $D$ if, according to every legitimate global measure of accuracy, $D$ is weakly-accuracy dominated by some credal state $D^{*}$, i.e., for every epistemically possible world, $D^{*}$ is just as accurate as $D$, and there exists at least one epistemically possible world $w^{*}$ in $W$ where $D^{*}$ is more accurate than $D .^{20}$

The Non-Dominance Principle underlies various central results of accuracy-centered epistemology, e.g., Joyce's $(1998,2009)$ argument for Probabilism and Briggs and Pettigrew's (forthcoming) argument for Bayesian conditionalization. As we understand the matter, the Non-Dominance Principle is a minimal commitment about epistemic rationality that any accuracy-centered epistemologist should take on board. So, if there are indeed imprecise credal states that are ruled permissible by Permission for Imprecision Precisified but ruled impermissible by the Non-Dominance Principle, then Permission for Imprecision Precisified cannot be accommodated within accuracy-centered epistemology.

The second principle we want introduce is:

The Joycean Constraint. For any partition $X$ over $W$, if $G_{X}$ is a legitimate global measure of accuracy for individual credence functions defined over $X$, then, for any probabilistically incoherent credence function $b$ defined over $X$, there is a probability function $p$ that weakly accuracy-dominates it, i.e., for every world $w$ in $W, G_{X}(b, w) \leq G_{X}(p, w)$ and there exists a world $w^{*}$ in $W, G_{X}\left(b, w^{*}\right)<$ $G_{X}\left(p, w^{*}\right)$.

We call this the Joycean constraint, since Joyce $(1998,2009)$ proves this claim from other assumptions about legitimate accuracy measures in his attempt to justify Probabilism by appealing to accuracy-based considerations. ${ }^{21}$ The Joycean Constraint and the Non-Dominance Principle together imply that if an agent has a precise credal state, then that doxastic state is rationally permissible only if it is representable by a probability function. This gives us a partial defense of Probabilism. 
Using the Joycean Constraint, we can now show that there are certain imprecise credal states deemed rational by Permission for Imprecision Precisified that violate the Non-Dominance Principle. Consider the following two cases:

FOUR-SIDED MYSTERY DIE. You have a four-sided die that was made at a factory where they can make dice of pretty much any bias. You've seen dice that always land on the same side, dice that always land on two sides with a frequency ratio 2:1 and never on the other two sides, etc. You have no idea whatsoever what bias your particular die has. What should your credence be that when you roll the die, it will land with side $<1>$ up?

FIVE-SIDED MYSTERY DIE. You have a five-sided die that was made at a factory where they can make dice of pretty much any bias. You've seen dice that always land on the same side, dice that always land on two sides with a frequency ratio 2:1 and never on the other three sides, etc. You have no idea whatsoever what bias your particular die has. What should your credence be that when you roll the die, it will land with side $<1>$ up?

Suppose your credences in these cases are defined over the partitions $X=$ $\left\{P_{1}, P_{2}, P_{3}, P_{4}\right\}$ and $X^{*}=\left\{P_{1}{ }^{*}, P_{2}{ }^{*}, P_{3}{ }^{*}, P_{4}{ }^{*}, P_{5}{ }^{*}\right\}$ respectively, where any $P_{i}$ is the proposition that the four-sided die lands with side $<i>$ facing up, and $P_{i}^{*}$ is the proposition that the five-sided die lands with side $<i>$ facing up. Since you have no idea about the bias of the die, Permission for Imprecision Precisified entails that it is rationally permissible for you in FOUR-SIDED MYSTERY DIE to adopt an imprecise credal state represented by the set of probability functions $C$ such that, for any $P$ in $X, C(P)=[0,1]$. Similarly, in FIVE-SIDED MYSTERY DIE, it is rationally permissible for you to adopt a doxastic state represented by the set of probability functions $C^{*}$ such that, for any $P$ in $X^{*}, C^{*}(P)=[0,1]$.

Now the main idea is this (for details, see the proof of Proposition 3 in the Appendix). According to Proposition 2, Local Extension, Local Boundedness, LocalGlobal Connection, and Local Admissibility guarantee that there is a precise credence function $p$ such that the credences it assigns to cells in $X$ are just as locally accurate as the imprecise credal assignments made by $C$. Likewise, we can construct a precise credence function $p^{*}$ such that the credences it assigns to the propositions in $X^{*}$ are just as accurate as the credal assignments made by $C^{*}$. But it will turn out that either $p$ or $p^{*}$ must be probabilistically incoherent. Then, by the Joycean Constraint, either $p$ or $p^{*}$ is weakly accuracy-dominated. But since $p$ is as accurate as $C$ in every possible world, and $p^{*}$ is as accurate as $C^{*}$ in every possible world, this means that either $C$ or $C^{*}$ is weakly accuracy-dominated. Thus, it seems that if the Joycean Constraint is true, some imprecise doxastic states that are allowed by Permission for Imprecision Precisified violate the Non-Dominance Principle and hence are irrational. Thus, Permission for Imprecision Precisified is false.

But note that the considerations raised in the preceding paragraph actually show something stronger: namely, certain probabilistic imprecise credal states will be accuracy-dominated given our constraints on accuracy measures, and will therefore violate Probabilistic Admissibility. So, in effect, the following impossibility result holds: 
Proposition 3. If Extension, Local Extension, Local Boundedness, Local Admissibility, Local-Global Connection, and Probabilistic Admissibility are true, then the Joycean Constraint is false.

\subsection{Summary}

Let's sum up. In this section, we have shown that given certain plausible constraints on local measures of accuracy, the accuracy-centered epistemologist can accept neither Weak Requirement of Imprecision nor the most natural precisification of Permission for Imprecision. If Weak Requirement of Imprecision and Permission for Imprecision were true, then imprecise credal states would be rationally permissible to adopt in any situation involving non-specific evidence, and might sometimes even be required. However, there's no easy way of reconciling this thesis with accuracycentered epistemology.

Note that the defender of imprecise credences cannot get out of this problem by retreating to the position that some other precisification of Permission for Imprecision is true. This is because the impossibility result given by Proposition 3 poses a dilemma for the accuracy-centered epistemologist who also wants to defend the rationality of imprecise credences. It shows that if Extension, Local Extension, Local Boundedness, Local-Global Connection and Local Admissibility are correct, then she must either reject the Joycean Constraint or Probabilistic Admissibility. On the one hand, since the accuracy-centered epistemologist needs the Joycean Constraint for accuracy-dominance arguments for Probabilism, she cannot reject it without non-trivial costs. On the other hand, Probabilistic Admissibility is attractive for the reasons discussed above. Therefore, the upshot of our argument is just that if our constraints on legitimate measures of accuracy are correct, then there is no satisfactory method of measuring the accuracy of imprecise credal states. This is why the consequences of Proposition 3 are much more serious than that of any other results that we have proved. ${ }^{22}$ In the remainder of this paper, we will discuss a few possible responses to our argument.

\section{Response 1: Adopting Non-Numerical Measures of Accuracy}

Throughout this paper, we have focused on a framework of accuracy-centered epistemology which makes use of numerical measures of accuracy, i.e., measures of accuracy that assign real values to credal states or credal assignments to propositions. This is precisely why we required that legitimate global and local measures of accuracy satisfy Boundedness and Local Boundedness, which entail that both global and local measures of accuracy should output real values. One may reject this constraint on measures of accuracy. A natural motivation for having nonnumerical measures of accuracy-which Mayo-Wilson and Wheeler (2016) attribute to James Joyce-is this. One might argue that when an agent's doxastic attitude towards a proposition is not representable by a real number, then the accuracy of her credal assignment should similarly not be representable by a real number. ${ }^{23}$ Since the proofs of Propositions $\mathbf{2}$ and $\mathbf{3}$ depend on Boundedness and Local Boundedness, 
using non-numerical measures of accuracy can help us block result like Propositions $\mathbf{2}$ and $\mathbf{3}$.

However, simply using non-numerical measures of accuracy does not by itself help us reconcile the rationality of imprecise credences with the accuracy-centered approach to epistemology. This is because a similar (though slightly weaker) result than Proposition 2 can also be proved for non-numerical measures of accuracy.

We begin with two prima facie attractive constraints on non-numerical measures of accuracy: Strong Extensionality and Completeness. Consider Strong Extensionality. To state this constraint precisely, we need to introduce the notion of an accuracy profile. Suppose $C$ is a set of credence functions defined over a partition $X$. The accuracy profile of $C$ at a world $w$ is the following multiset:

$$
\left\{\left\{\left(v_{w}(P), C(P)\right): P \in X\right\}\right\}^{24}
$$

In other words, the accuracy profile of $C$ at $w$ contains, for each proposition in $X$, the pair consisting of the truth value of that proposition at $w$, and the set of credences that $C$ assigns to that proposition. Strong Extensionality says that the accuracy of $C$ at $w$ is determined entirely by the accuracy profile of $C$ at $w$. That is:

Strong Extensionality. For any partition $X$ over a set of worlds $W$, let $C$ and $C^{*}$ be sets of credence functions in $B_{X}$. If $G_{X} *$ is a legitimate imprecise global measure of accuracy, and the accuracy profile of $C$ at $w$ in $W$ is the same as the accuracy profile of $C^{*}$ at $w^{*}$ in $W$, then $G_{X} *(C, w)=G_{X} *\left(C^{*}, w^{*}\right){ }^{25}$

The rationale for Strong Extensionality is that the accuracy of a credal state at a world should only depend on (i) the truth values at that world of the propositions over which the credal state is defined and (ii) the values that the credal state assigns to those propositions. To reject Strong Extensionality is to allow measures of accuracy to be sensitive to factors other than truth-values of propositions and the relevant credal assignments, e.g., factors like the informativeness of the propositions. But why should a measure that assesses solely the accuracy of credal states and not something else be sensitive to such factors? Thus, rejecting Strong Extensionality is a bad idea.

The second constraint-Completeness-is more questionable: it says that any legitimate accuracy measure for imprecise credal states must be such that every credal state should be comparable with respect to global accuracy with all other credal states.

Completeness. For any partition $X$ over a set of worlds $W$, let $C$ and $C^{*}$ be sets of credence functions in $B_{X}$. If $G_{X} *$ is a legitimate imprecise global measure of accuracy, then, for any $w$ in $W$, either $G_{X} *(C, w)>G_{X}{ }^{*}\left(C^{*}, w\right)$ or $G_{X} *(C, w)<$ $G_{X} *\left(C^{*}, w\right)$ or $G_{X} *(C, w)=G_{X} *\left(C^{*}, w\right){ }^{26}$

Note that Completeness trivially follows from Boundedness (since it would be true whenever $G_{X}{ }^{*}$ assigns real values), but it is also much weaker. According to Completeness, any legitimate measure of accuracy should be such that the global accuracy of one credal state under that rule must either be greater than, less than, 
or equal to the global accuracy of another credal state. Now, this constraint may indeed be true of non-numerical measures of accuracy.

With these two constraints in place, we can prove the following claim (which generalizes another result proved by Schoenfield (2015)).

Proposition 4. For any finite partition $\mathrm{X}$ over a set of worlds $W$, let $\mathrm{C}$ and $\mathrm{C}^{*}$ be sets of probability functions in $\mathrm{B}_{\mathrm{X}}$, such that for any $\mathrm{P}$ in $\mathrm{X}$ and some set of real numbers $\mathrm{R}, \mathrm{C}(\mathrm{P})=\mathrm{R}$ and $\mathrm{C}^{*}(\mathrm{P})=\{1 /|\mathrm{X}|\}$. If Strong Extensionality, Completeness, and Probabilistic Admissibility are true, then, for any legitimate imprecise global measure of accuracy $\mathrm{G}_{\mathrm{X}} *$ and any world $\mathrm{w}$ in $\mathrm{W}, \mathrm{G}_{\mathrm{X}} *(\mathrm{C}, \mathrm{w})=\mathrm{G}_{\mathrm{X}} *\left(\mathrm{C}^{*}, \mathrm{w}\right)$.

Let's see what this means. Consider the Principle of Indifference, i.e., the principle that for any finite partition $\mathrm{X}$ over a set of worlds $\mathrm{W}$, an agent's credence in any $P$ in $X$ (in the absence of evidence that favours any particular $\mathrm{P}$ ) should be $1 /|\mathrm{X}|$. According to Proposition 4, any imprecise credal state that assigns an interval $[a, b]$ to every cell of a finite partition is just as accurate as the precise credal state recommended by the Principle of Indifference with respect to that partition. For instance, take the case where $X=\{F H, F T, B H, B T\}$. If our evidence is maximally unspecific about the bias of the coin, the same line of reasoning that motivates Requirement of Imprecision should also require the agent to adopt an imprecise credal state that assigns [0,1] to each cell of this extended partition. ${ }^{27}$ But Proposition 4, in conjunction with other assumptions, shows that this is false. For if Completeness, Strong Extensionality, and Probabilistic Admissibility are true, then the credal state that is mandated by the Principle of Indifference (and therefore assigns $1 / 4$ to each cell in the partition) is just as accurate as the imprecise credal state in every world. And if the Permission Principle is true, then the agent cannot be required to adopt either of these two credal states in any world. This immediately undermines some of the motivation for both Requirement of Imprecision and Weak Requirement of Imprecision.

Even though Proposition 4 (unlike Propositions 2 and 3) doesn't straightforwardly refute Weak Requirement of Imprecision or Permission for Imprecision, it poses a problem for Weak Requirement of Imprecision. ${ }^{28}$ In conjunction with Completeness, Strong Extensionality, Probabilistic Admissibility, and the Permission Principle, Proposition 4 implies that an agent can never be required to adopt an imprecise credal state that assigns the same set of credences, e.g., an interval $[a, b]$, to every cell of the partition. Call such imprecise credal states uniform. Proposition 4 (along with the other constraints) entails that if an agent is ever required to adopt an imprecise credal state in response to a body of non-specific evidence, the relevant credal state must be non-uniform. If the defender of Weak Requirement of Imprecision accepts this claim, she must explain why uniform imprecise credal states should be treated differently from non-uniform imprecise credal states. In any plausible case of nonspecific evidence, what seems to make an imprecise credal state rationally required is just the fact that the agent has no information about the propositions contained in the relevant partition, except for the information that the chance distribution over that partition lies within a certain set of probability distributions. As long as that set of probability distributions assigns the same set of real numbers to each 
cell of the partition, we will continue to have the intuition that the agent is required to adopt a uniform imprecise credal state in that situation. Thus, it just seems ad hoc to claim that there can be situations of non-specific evidence where an agent is required to adopt non-uniform credal states, but there can't be situations of nonspecific evidence where she is required to adopt uniform credal states. In order to make her account less ad hoc, the defender of imprecise credences would have to reject Weak Requirement of Imprecision.

The defender of non-numerical measures of accuracy could block this argument by rejecting either Completeness, or Strong Extensionality, or Probabilistic Admissibility. For now, let's set aside the question of rejecting Probabilistic Admissibility: while rejecting Probabilistic Admissibility would block all the results we have proved so far, it is far from clear whether rejecting it will help the defender of imprecise credences in this context. ${ }^{29}$ Now, the non-numerical accuracy measures that have been proposed in the literature on imprecise credences - e.g., the one proposed by Seidenfeld, Schervish and Kadane (2012) and another ascribed to James Joyce by Mayo-Wilson and Wheeler (2016) -violate either Completeness or Strong Extensionality (see Appendix B). We have already explained why rejecting Strong Extensionality is a bad idea. So, the only remaining option for the defender of imprecise credences would be to adopt measures of accuracy that violate Completeness.

We think rejecting Completeness will not help the defender of Weak Requirement of Imprecision. Consider two different probabilistic credal states, represented by the sets of credence functions $C_{1}$ and $C_{2}$ defined over an $n$-cell partition $X$, such that for any $P$ in $X, C_{1}(P)=[a, b]$ and $C_{2}(P)=\{1 / n\}$. There are two options: either the defender of Weak Requirement of Imprecision could say that an agent cannot ever be in a situation of non-specific evidence where adopting the credal state corresponding to $C_{1}$ is required by rationality, or she could deny this. The first option, as we have argued, makes the defense of Weak Requirement of Imprecision $\mathrm{ad}$ hoc. The second option involves saying that an agent can be required by rationality to adopt the imprecise credal state represented by $C_{1}$. But note that if the defender of Weak Requirement of Imprecision wants to avoid the consequences of Proposition 4 by denying Completeness, she would have to say that the imprecise credal state represented by $C_{1}$ is incomparable with respect to global accuracy to the precise credal state represented by $C_{2}$ in every possible world. ${ }^{30}$ Now, plausibly, if two credal states are incomparable to each other with respect to global accuracy in every possible world, then a rational agent cannot regard any one of the credal states as better than, worse than, or exactly as good as the other given solely the metric of global accuracy. Then, given solely considerations about global accuracy, an agent also cannot have any reason to adopt the imprecise credal state over the precise one.

This raises a challenge for the defender of Weak Requirement of Imprecision. If she wants to say that an agent can be required by rationality to adopt the imprecise credal state represented by $C_{1}$, she must explain, without appealing to the global accuracy of that credal state, how there could be an accuracy-based advantage to adopting that credal state. The most natural explanation would be that the imprecise credal state matches the specificity of the agent's evidence, while 
the precise credal state doesn't. But this explanation is not satisfying: it's unclear why it is better from an accuracy-centered standpoint to match one's credal state to the specificity of one's evidence. In the absence of a good explanation, Weak Requirement of Imprecision cannot be saved by rejecting Completeness.

Our argument in this section leaves open the possibility that Permission for Imprecision can be true from an accuracy-centered standpoint. Therefore, one consequence of our argument is that a defender of imprecise credences who adopts the strategy of adopting non-numerical accuracy measures can only plausibly accept Permission for Imprecision but not Weak Requirement of Imprecision. Since a view of this kind seems quite weak, the defender of imprecise credences might ultimately have to seek a different way of defending the rationality of imprecise credences from an accuracy-centered standpoint.

\section{Response 2: Rejecting Probabilistic Admissibility}

The consequences of Proposition 3 are far more serious than that of any other results that we have proved so far. As we pointed out above, it shows that given our constraints on accuracy measures for imprecise credences, there is no measure of accuracy that respects both the Joycean Constraint and Probabilistic Admissibility. Since both these principles are attractive from an accuracy-centered standpoint, this just shows that there is no satisfactory method of measuring the accuracy of imprecise credal states using numerical accuracy measures. In this section, we will explore whether a defender of imprecise credences can avoid this consequence by rejecting Probabilistic Admissibility.

Interestingly, some have in fact been tempted to reject Probabilistic Admissibility. For instance, Konek (forthcoming) proposes a global accuracy measure which violates this constraint (see Appendix C). Note that, for the accuracy-centered epistemologist, rejecting Probabilistic Admissibility outright is a bad idea, since much of the work done in accuracy-centered epistemology depends on the idea that precise probability functions shouldn't be accuracy-dominated. But perhaps she can adopt a restricted version of Probabilistic Admissibility instead, such as:

Restricted Probabilistic Admissibility. For any set of worlds $W$ and a partition $X$ over $W$, if $G_{X}$ * is a legitimate imprecise global accuracy measure for sets of credence functions defined over $X$, then for any probability function $p$ in $B_{X}$, there is no set $C$ of credence functions in $B_{X}$ such that $p$ is accuracy-dominated by $C$ according to $G_{X}$ *.

In other words, there is no set $C$ in $B_{X}$ such that

(i) for every world $w$ in $W, G_{X} *(\{p\}, w) \leq G_{X} *(C, w)$, and

(ii) there exists a world $w$ in $W$ such that $G_{X} *(\{p\}, w)<G_{X} *(C, w)$.

Restricted Probabilistic Admissibility preserves the idea that precise credence functions shouldn't be accuracy-dominated, but it nevertheless avoids the impossibility result from the preceding section. Importantly, Konek's global measure of accuracy violates not only Probabilistic Admissibility, but also Restricted Probabilistic 
Admissibility (see Appendix C). However, even if it were possible to formulate accuracy measures for imprecise credal states that preserve Restricted Probabilistic Admissibility, adopting such a weak principle still seems highly problematic to us.

Now, it may be possible to reconcile Permission for Imprecision with accuracycentered epistemology by retreating to Restricted Probabilistic Admissibility. However, this strategy might be reasonable if only a handful of imprecise credal states were accuracy-dominated in the way Proposition 3 suggests. In such a case, the defender of imprecise credences might still be able to hold that in most but not all cases of non-specific evidence, an agent is permitted to have imprecise credences. But we think that on the view proposed here, it is highly likely that not just some but most imprecise credal states are accuracy-dominated by some precise credence function. In order to see this, note that the proof of Proposition $\mathbf{2}$ is a proof by construction. More specifically, it proceeds by constructing a precise credence function that is just as accurate as the imprecise credal state by considering in isolation the appropriate credence that the precise function needs to assign to each proposition in the relevant partition, so that both the precise and the imprecise states have the same local accuracy score with respect to each proposition (and then Local-Global Connection guarantees that this function will be just as accurate globally). But there is nothing in this procedure that guarantees that the precise credence function will be probabilistically coherent; for it may well be that all the individual credal assignments that the precise function assigns to different propositions in the partition don't add up to exactly 1 .

One last point: the proof of Proposition 3 shows that if we accept certain constraints on legitimate accuracy measures for imprecise credal states and the Joycean Constraint, some probabilistic imprecise credal states that seem rational in light of Permission for Imprecision will end up being accuracy-dominated. Now, the accuracy-centered epistemologist may reject Probabilistic Admissibility and accept Restricted Probabilistic Admissibility on the ground that only a small number of probabilistic imprecise doxastic states end up being accuracy-dominated on the resulting view. Still, a problem will persist; for the imprecise credal states that are ruled out by our constraints look just like all the other credal assignments the defender of imprecise credences endorses. Thus, it seems that if this is the route that the accuracy-centered epistemologist wants to go, she owes us an independent story as to which imprecise credal states are irrational, and which are not. Otherwise the kinds of credal assignments the defender of imprecise credences will endorse will turn out to be hopelessly unsystematic.

\section{Response 3: Rejecting Local-Global Connection}

A promising strategy for blocking the arguments for both Propositions 2 and 3 would be to reject Local-Global Connection. ${ }^{31}$ Local-Global Connection involves two constraints. According to the first, for any legitimate global accuracy measure, there exists a legitimate local accuracy measure such that if two credal states make equally accurate credal assignments to each proposition in a partition according to the local measure, then the global accuracy of the two states is the same according 
to the global measure. According to the second, for any legitimate local accuracy measure, there exists a legitimate global accuracy measure, such that if two credal states make equally accurate credal assignments to each proposition in a partition according to the local measure, then the global accuracy of the two states is the same according to the global measure.

Local-Global Connection can lead to problems. We might think that even if the credal assignments made by two imprecise credal states have the same local accuracy scores, the global accuracy score of the two states might still differ. Suppose $C$ is a set of probability functions such that, for any $P$ in $X=\{H, T\}, C(P)=[0.2,0.8]$. Now, take another set of credence functions $C^{*}$ which contains all the probability functions in $C$ but contains one probabilistically incoherent function $b$ such that, for any $P$ in $X, b(P)=0.8$. But note that for any $P$ in $X, C(P)=C^{*}(P)$. Therefore, by Local-Global Connection, the global accuracy of $C$ and $C^{*}$ ought to be the same. This might seem like a bad consequence of Local-Global Connection: if $C$ and $C^{*}$ have the same global accuracy score, there is no hope for offering an accuracydominance argument for Probabilism with respect to imprecise credal states (i.e., for a view that all the credence functions in one's representor have to be probability functions). This might lead us to reject Local-Global Connection. If we reject it, we are able to block Proposition 2 and Proposition 3.

We have two counter-responses. First of all, even if the defender of imprecise credences rejects Local-Global Connection, it won't help her block Proposition 4. She will end up rejecting the Weak Requirement of Imprecision (as shown in §5) as long as she accepts Completeness, Strong Extensionality, and Probabilistic Admissibility.

Second, Local-Global Connection still seems independently appealing. It's motivated by the natural thought that the global accuracy of a doxastic state (which involves doxastic attitudes towards different propositions) should depend only on the local accuracy of the doxastic attitudes that it involves. So, if there are two doxastic states which involve doxastic attitudes that match each other perfectly with respect to local accuracy, then the two doxastic states should not be more or less globally accurate than each other. So far, we have had no reason to think that this principle should fail with respect to precise credal states or with respect to imprecise probabilistic credal states. This might give us reason to think that the principle is true across the board. This in turn might give us reason to search for a different solution to the problem mentioned above. Here is a sketch of such a solution.

In response to the problem raised above, we ought to concede that when it comes to imprecise credal states, there is no straightforward accuracy-dominance argument for Probabilism. This, however, doesn't mean that there is no accuracybased reason for ruling out non-probabilistic credal states like the one represented by $C^{*}$. It seems to us that a defender of imprecise credences should accept the following principle on independent grounds:

Rational Admissibility. An agent is rationally permitted to adopt an imprecise credal state represented by a set $C$ of credence functions only if $C$ is a rationally admissible set of credence functions, i.e., for any credence function $b$ in $C$, there 
is some epistemic situation in which it is rationally permissible for an agent to adopt $b$.

This requirement should be intuitively attractive for the defender of imprecise credences. She should want the credence functions in the representor to be such that, on receiving more information, it can be rationally permissible for the agent to adopt each credence function in the representor as her actual credence function. For example, in MYSTERY COIN, the only credence functions that should be in your representor ought to be those that you would be permitted to adopt on gaining more information about the bias of the coin. If this requirement is correct, it will be enough to rule out the possibility that the non-probabilistic credal states (represented by sets of credence functions some of which may be probabilistically incoherent) are ever rationally permissible to adopt. Joyce's (1998, 2009) accuracydominance argument shows that probabilistically incoherent credence functions are never rationally permissible to adopt, because they are accuracy-dominated by some other, coherent function. So, a set of credence functions that contains probabilistically incoherent credence functions will be rationally inadmissible, and therefore a credal state represented by such a set of credence functions will be ruled out by Rational Admissibility.

An apparent cost of Rational Admissibility is that it conflicts with the Permission Principle. We are saying that an agent may be rationally required to adopt the probabilistic credal state represented by $C$ over the non-probabilistic credal state represented by $C^{*}$ even though the global accuracy of $C$ and $C^{*}$ is the same. Given that we ourselves have relied on the Permission Principle in our argument against Weak Requirement of Imprecision given in $\$ 4.1$, the strategy of accepting Rational Admissibility seems to undermine our own argument. However, this may in fact indicate that the accuracy-centered epistemologist should accept a different version of the Permission Principle that is restricted to rationally admissible doxastic states. As far as we can tell, adopting this more restricted version of the Permission Principle doesn't affect our argument against Weak Requirement of Imprecision. This is because, for any imprecise probabilistic credal state, Proposition 2 guarantees that, given our constraints on measures of accuracy, there is another precise credal state that is just as accurate as the imprecise one. Now, there are two possibilities: either that precise credal state is probabilistically coherent, or it's not. If it is probabilistically coherent, then the restricted version of the Permission Principle says that the agent isn't required to have that imprecise probabilistic state. If it is not probabilistically coherent, then by the Joycean Constraint, there is a precise probabilistic credal state that accuracy-dominates it, and therefore accuracy-dominates the imprecise credal state. In that case, too, the agent isn't required to have that imprecise probabilistic state. In either case, Weak Requirement of Imprecision fails.

The upshot is this. While it might be tempting for the defender of imprecise credences to reject Local-Global Connection in light of certain problems, this isn't obviously a viable option. On the one hand, she will still have to deal with the problems that arose from Proposition 4. On the other hand, given the independent appeal of Local-Global Connection, other solutions to the relevant problems may 
also be worth pursuing. Moreover, if the defender of imprecise credences were able to show that our solution from Rational Admissibility doesn't work and that LocalGlobal Connection is ultimately indefensible, that would in itself be a significant achievement. For it would show that certain initially attractive constraints, like Local-Global Connection, which accuracy-centered epistemologists happily accept when it comes to precise credal states, cannot be reconciled at all with the rationality of imprecise credences.

\section{Conclusion}

Let us take stock. A central motivation for imprecise credences comes from situations involving non-specific evidence: for instance, when an agent has no information whatsoever about the bias of a coin, it seems that she is permitted, and perhaps even required, not to assign any sharp credence to the proposition that the coin will land heads (or tails) when flipped. Some take such scenarios to show that for any scenario where an agent's total evidence is non-specific, she is required by rationality to adopt imprecise credences. We called this view Requirement of Imprecision.

We began this essay with Schoenfield's argument against Requirement of Imprecision. She shows that at least for imprecise probabilistic credal states defined over two-cell partitions, there is always a precise probabilistic credal state which is just as accurate as the imprecise state in every world. This shows that accuracy-centered epistemologists cannot accommodate Requirement of Imprecision. However, we showed that even if the defender of imprecise credences rejects Requirement of Imprecision in light of Schoenfield's argument, she may still be able to allow imprecise credal states to be permissible in all situations of nonspecific evidence, and required in some of them. In response, we argued that if we accept some other plausible constraints on accuracy measures for imprecise credal states, this possibility is also blocked. Finally, we considered a number of responses to our argument, each of which involved rejecting one or more of the constraints that we imposed on measures of accuracy. We went on to argue that each of these responses involve some intuitive or theoretical costs. The upshot of our argument, therefore, is that given our constraints, there is no plausible way of measuring the accuracy of imprecise credal states; so, the rationality of imprecise credences cannot easily be accommodated within accuracy-centered epistemology.

\section{Appendix A: Proofs}

Proposition 1. For any set of worlds $\mathrm{W}$ and a two-cell partition $\mathrm{X}$ over $\mathrm{W}$, let $\mathrm{C}$ be a set of probability functions in $\mathrm{B}_{\mathrm{X}}$ (the set of credence functions defined over X). Then, if Extension, Boundedness, Probabilistic Admissibility, and WorldInvariance are true, then, for any legitimate imprecise global accuracy measure $\mathrm{G}_{\mathrm{X}}$ *, there will be a probability function $\mathrm{b}$ in $\mathrm{B}_{\mathrm{X}}$ such that, for any $\mathrm{w}$ in $\mathrm{W}, \mathrm{G}_{\mathrm{X}} *(\mathrm{C}, \mathrm{w})=$ $\mathrm{G}_{\mathrm{X}} *(\{\mathrm{~b}\}, \mathrm{w})$. 
To prove Proposition 1, we need:

Lemma 1. For any set of worlds $W$, let $\mathrm{X}=\{\mathrm{H}, \mathrm{T}\}$ be a partition over $\mathrm{W}$. Then, if Probabilistic Admissibility and World-Invariance are true, for any legitimate imprecise global accuracy measure $\mathrm{G}_{\mathrm{X}} *$, if $\mathrm{C}$ and $\mathrm{C}^{*}$ are sets of probability functions defined over $\mathrm{X}$, the following conditional holds:

If (i) for any $H$-world $w, G_{X} *(C, w)=G_{X} *\left(C^{*}, w\right)$, then (ii) for any $T$-world $w^{*}$, $G_{X} *\left(C, w^{*}\right)=G_{X} *\left(C^{*}, w^{*}\right)$.

Proof of Lemma 1. Suppose the conditional is false: (i) is true, but (ii) isn't. Then, there exists a $T$-world $w^{*}$ s.t. either $G_{X} *\left(C, w^{*}\right)>G_{X} *\left(C^{*}, w^{*}\right)$ or $G_{X} *\left(C, w^{*}\right)<$ $G_{X} *\left(C^{*}, w^{*}\right)$. But note that by World-Invariance, if for a $T$-world $w^{*}, G_{X} *\left(C, w^{*}\right)$ $>G_{X} *\left(C^{*}, w^{*}\right)$, then, for every $T$-world $w^{* *}, G_{X} *\left(C, w^{* *}\right)>G_{X} *\left(C^{*}, w^{* *}\right)$. In that case, since (i) is true, $C^{*}$ will be weakly accuracy-dominated and therefore will violate Probabilistic Admissibility. A similar violation of Probabilistic Admissibility will occur if, for a $T$-world $w^{*}, G_{X} *\left(C, w^{*}\right)<G_{X} *\left(C^{*}, w^{*}\right)$. Therefore, (ii) must be true.

Proof of Proposition 1. Suppose an imprecise doxastic state, defined over a partition $X=\{H, T\}$, is represented by a set $C$ of probability functions in $B_{X}$. By Boundedness, for any $H$-world $w, G_{X} *(C, w)$ is between 0 and 1 . So by the continuity of $G_{X}$ over the space of probability functions, there is some probability function $b$ in $B_{X}$ such that $G_{X} *(C, w)=G_{X}(b, w)$. (This follows from the intermediate value theorem.) By Extension, it follows that $G_{X} *(C, w)=G_{X} *(\{b\}, w)$. By World-Invariance, it follows that for every $H$-world $w^{*}, G_{X} *\left(C, w^{*}\right)=G_{X} *\left(\{b\}, w^{*}\right)$. Combining this with Lemma 1, we get that for every $T$-world $w^{* *}, G_{X} *\left(C, w^{* *}\right)=G_{X} *\left(\{b\}, w^{* *}\right)$. Thus, Proposition 1 is proved.

Proposition 2. Suppose an imprecise credal state, defined over a partition $\mathrm{X}$ over a set of worlds $\mathrm{W}$, is represented by a set $\mathrm{C}$ of functions in $\mathrm{B}_{\mathrm{X}}$. If Local Extension, Local Boundedness, Local Admissibility, and Local-Global Connection are true, then there will be a credence function $\mathrm{b}$ in $\mathrm{B}_{\mathrm{X}}$ such that for any legitimate imprecise global measure of accuracy $\mathrm{G}_{\mathrm{X}} * \mathrm{G}_{\mathrm{X}} *(\mathrm{C}, \mathrm{w})=\mathrm{G}_{\mathrm{X}} *(\{\mathrm{~b}\}, \mathrm{w})$ in every world $\mathrm{w}$.

To prove Proposition 2, we prove:

Lemma 2. Suppose $\mathrm{R}$ is a set of real numbers between 0 and 1 (inclusive), and $\mathrm{c}$ is a real number between 0 and 1 (inclusive). If Local Boundedness, Local Extension, and Local Admissibility are true, then, for any legitimate local measure of accuracy $\mathrm{L}^{*}, \mathrm{~L}^{*}(\mathrm{R}, 1)=\mathrm{L}^{*}(\{\mathrm{c}\}, 1)$ iff $\mathrm{L}^{*}(\mathrm{R}, 0)=\mathrm{L}^{*}(\{\mathrm{c}\}, 0)$.

Proof of Lemma 2. Now, suppose $L^{*}(R, 1)=L^{*}(\{c\}, 1)$, but either (i) $L^{*}(R, 0)$ $>L^{*}(\{c\}, 0)$ or (ii) $L^{*}(R, 0)<L^{*}(\{c\}, 0)$. Let (i) be true. By Local Boundedness, $L^{*}(R, 0)$ is between 1 and 0 (inclusive). Since $L(., 0)$ is continuous through the unit interval $[0,1]$, we can find a real number $d$ between 0 and 1 such that $L^{*}(R, 0)=$ $L(d, 0)=L^{*}(\{d\}, 0)$ (once again, by the intermediate value theorem). But note that if (i) is true, $d$ is distinct from $c$. We show that this cannot be the case. There are two possibilities. 
Possibility 1. Suppose $c<d$. Then, we pick a real number $r$ between $c$ and $d$ (exclusive). Then, by Truth-Directedness and Local Extension,

$$
\begin{aligned}
& L^{*}(R, 1)=L^{*}(\{c\}, 1)<L^{*}(\{r\}, 1), \\
& L^{*}(R, 0)=L^{*}(\{d\}, 0)<L^{*}(\{r\}, 0)
\end{aligned}
$$

But, since $\{r\}$ strongly accuracy-dominates $R$, we violate Local Admissibility.

Possibility 2. Suppose $c>d$. Then, we pick a real number $r$ between $d$ and $c$ (exclusive).Then, by Truth-Directedness,

$$
\begin{aligned}
& L^{*}(R, 1)=L^{*}(\{c\}, 1)>L^{*}(\{r\}, 1) . \\
& L^{*}(R, 0)=L^{*}(\{d\}, 0)>L^{*}(\{r\}, 0) .
\end{aligned}
$$

But, since $\{r\}$ is strongly accuracy-dominated by $R$, we violate Local Admissibility.

So, $c=d$. But in that case, (i) cannot be true. Similarly, (ii) cannot be true. The converse is proved exactly in the same way.

Proof of Proposition 2. Let $C$ be a set of probability functions defined over a partition $X$ over a set of worlds $W$. Let $L^{*}$ be an arbitrary legitimate local measure of accuracy.

Consider any world $w$ in $W$. For any proposition $P$ in $X$, if $w$ is in $P$, then the local accuracy of the credal assignment that $C$ makes to $P$ is $L^{*}(C(P), 1)$. By Local Extension, there exists a legitimate local accuracy measure $L$ of which $L^{*}$ is an extension. By Local Boundedness, $L^{*}(C(P), 1)$ is between 1 and 0 (inclusive). Since $L(., 1)$ is continuous through the unit interval $[0,1]$, we can find a real number $x$ between 0 and 1 (inclusive) such that $L(x, 1)=L^{*}(C(P), 1)$ (by the intermediate value theorem). Analogously, if $w$ is not in $P$, then the local accuracy of the credal assignment that $C$ makes to $P$ is $L^{*}(C(P), 0)$. By Local Boundedness, $L^{*}(C(P), 0)$ is between 1 and 0 . Since $L(., 0)$ is continuous through the unit interval $[0,1]$, we can find a real number $x$ between 0 and 1 such that $\mathrm{L}(x, 0)=L^{*}(C(P), 0)$ (by the intermediate value theorem). Therefore, for any $P$ in $X$, there exists a real number $x$ between 0 and 1 (inclusive) such that $L^{*}\left(C(P), v_{w}(P)\right)=L^{*}\left(\{x\}, v_{w}(P)\right)$. This entails that there exists a credence function $b$, such that for any $P$ in $X$, $L^{*}\left(C(P), v_{w}(P)\right)=L^{*}\left(\{b(P)\}, v_{w}(P)\right)$. In other words, there exists a credence function $b$ such that for any proposition $P$ in $X$, the credal assignment made by $b$ to $P$ is just as locally accurate in $w$ as the credal assignment made by $C$ to $P$.

Now, consider any world $w^{*}$ such that $w \neq w^{*}$. There are two possibilities: either $w$ and $w^{*}$ belong to the same cell of the partition $X$, or they don't.

Possibility 1. If $w$ and $w^{*}$ belong to the same cell of the partition $X$, then $w^{*}$ will assign the same truth-values to propositions in $X$ as $w$; so, for any $P$ in $X$, $v_{w}(P)=v_{w^{*}}(P)$. Hence, for any proposition $P$ and any credence function $b$ such that $L^{*}\left(C(P), v_{w}(P)\right)=L^{*}\left(\{b(P)\}, v_{w}(P)\right)$, it will be the case that $L^{*}(C(P)$, $\left.v_{w^{*}}(P)\right)=L^{*}\left(\{b(P)\}, v_{w^{*}}(P)\right)$. 
Possibility 2. If $w$ and $w^{*}$ don't belong to the same cell of the partition $X$, then there exist exactly two propositions $P$ and $P^{*}$ in $X$ such that $w$ is in $P$ but not in $P^{*}$, whereas $w^{*}$ is in $P^{*}$ but not in $P$. Therefore, $v_{w}(P) \neq v_{w^{*}}(P)$ and $v_{w}\left(P^{*}\right) \neq v_{w^{*}}\left(P^{*}\right)$. According to Lemma 2, however, for any sets of credences $R$ and $\{c\}, L^{*}(R, 1)=L^{*}(\{c\}, 1)$ iff $L^{*}(R, 0)=L^{*}(\{c\}, 0)$. So, whatever $P$ and $P^{*}$ may be, if $L^{*}\left(C(P), v_{w}(P)\right)=L^{*}\left(\{b(P)\}, v_{w}(P)\right)$ and $L^{*}\left(C\left(P^{*}\right), v_{w}\left(P^{*}\right)\right)$ $=L^{*}\left(\left\{b\left(P^{*}\right)\right\}, v_{w}\left(P^{*}\right)\right)$, then $L^{*}\left(C(P), v_{w^{*}}(P)\right)=L^{*}\left(\{b(P)\}, v_{w^{*}}(P)\right)$ and $L^{*}\left(C\left(P^{*}\right), v_{w^{*}}\left(P^{*}\right)\right)=L^{*}\left(\left\{b\left(P^{*}\right)\right\}, v_{w^{*}}\left(P^{*}\right)\right)$.

This entails that for any $w$ in $W$ and any $P$ in $X, L^{*}\left(C(P), v_{w}(P)\right)=L^{*}\left(\{b(P)\}, v_{w}(P)\right)$. Since $L^{*}$ is any arbitrary legitimate local accuracy measure, by Local-Global Connection, it follows that for any legitimate global measure of accuracy $G_{X}{ }^{*}, G_{X} *(C, w)$ $=G_{X} *(\{b\}, w)$.

Proposition 3. If Extension, Local Extension, Local Boundedness, Local Admissibility, Local-Global Connection, and Probabilistic Admissibility are true, then the Joycean Constraint is false.

Proof of Proposition 3. We prove this proposition for the general case. Let $X=$ $\left\{P_{1}, \ldots, P_{n}\right\}$ and $X^{*}=\left\{P_{1}^{*}, \ldots, P_{n+1} *\right\}$, both defined over the same set of worlds $W$. Let $C$ represent a probabilistic imprecise doxastic state defined over $X$, such that for any $P$ in $\mathrm{X}, C(P)=[0,1]$. Similarly, let $C^{*}$ represent a probabilistic imprecise credal state defined over $X^{*}$, such that for any $P$ in $X^{*}, C^{*}(P)=[0,1]$.

By the proof of Proposition 2, if Local Extension, Local Boundedness, and Local Admissibility are true, we can show that for any legitimate local measure of accuracy $L^{*}$, there exists a precise credence function $b$ such that for any $w$ in $W$ and any proposition $P$ in $X, L^{*}\left(C(P), v_{w}(P)\right)=L^{*}\left(\{b(P)\}, v_{w}(P)\right)$ for all propositions $P$ in $X$. Suppose, now, we construct a credence function $b^{*}$ such that for any $P_{i}$ in $X$ and $P_{i}{ }^{*}$ in $X^{*}$ where $i$ is between 1 and $n$ (inclusive), $b^{*}\left(P_{i}^{*}\right)=b\left(P_{i}\right)$. However, for the proposition $P_{n+1} *$, we let $b^{*}\left(P_{n+1} *\right)=r$ such that $L^{*}\left(C^{*}\left(P_{n+1} *\right), v_{w}\left(P_{n+1}{ }^{*}\right)\right)$ $=L^{*}\left(\{r\}, v_{w}\left(P_{n+1} *\right)\right)$ for all $w$ in $W$. That there will be such an $r$ is guaranteed by Local Extension, Local Boundedness, and Lemma 2. Note that due to Local-Global Connection, this guarantees that for any $w$ in $W$ and for any legitimate global measure of accuracy $G_{X^{*}}, G_{X^{*}} *\left(C^{*}, w\right)=G_{X^{*}} *\left(\left\{b^{*}\right\}, w\right)$.

Now, we show that we are bound to get a violation of Probabilistic Admissibility in this case if the Joycean Constraint is true.

Possibility 1. Suppose $b$ is a probability function; then $\sum_{P \in X} b(P)=1$. But now consider $\sum_{P \in X^{*}} b^{*}(P)$. Since $b^{*}$ assigns the same values to the first $n$ cells in $X^{*}$ as $b$ does to the first $n$ cells in $X$, we have $\sum_{P \in X^{*}} b^{*}(P)=\sum_{P \in X} b(P)+b^{*}\left(P_{n+1} *\right)=$ $1+r$. Here, there are two possibilities: either $r>0$ or $r=0$.

Possibility 1.1. If $r>0, b^{*}$ is probabilistically incoherent. Then, by the Joycean Constraint, according to any legitimate precise global accuracy measure $G_{X^{*}}$, there is a probability function that weakly accuracy-dominates $b^{*}$. By Extension, since $G_{X^{*}} *$ is an extension of a legitimate accuracy score $G_{X^{*}}$, according to $G_{X^{*}}{ }^{*}$, $C^{*}$ is accuracy-dominated by a precise credal state. Thus, we get a violation of Probabilistic Admissibility. 
Possibility 1.2. Now, consider the possibility that $r=0$. Let $L^{*}$ be any arbitrary local accuracy measure for imprecise credences. By Local Boundedness and Local Extension, $L^{*}(\{r\}, 1)=0$ and $L^{*}(\{r\}, 0)=1$. Now, since $C^{*}\left(P_{n+1} *\right)=[0,1]$ and the local accuracy score of $\{r\}$ and $C^{*}\left(P_{n+1}{ }^{*}\right)$ is the same relative to any truthvalue, $L^{*}([0,1], 0)=L^{*}(\{0\}, 0)=1$ and $L^{*}([0,1], 1)=L^{*}(\{0\}, 1)=0$. Consider the credence function $b^{* *}$ such that for any $P$ in $X^{*}, b^{* *}(P)=0$. Since for any $P$ in $X^{*}, C^{*}(P)=[0,1]$, this means that for any $P$ in $X^{*}$, the local accuracy score of $\left\{b^{* *}(P)\right\}$ and $C^{*}(P)$ will be the same relative to any truth-value. By Local-Global Connection, this implies that for any $w$ in $W, G_{X^{*}} *\left(C^{*}, w\right)=G_{X^{*}}\left(\left\{b^{* *}\right\}, w\right)$. Since $b^{* *}$ is not a probability function, using the Joycean Constraint, we get the result that there exists a probability function that weakly accuracy-dominates $b^{* *}$. This means that $C^{*}$ is accuracy-dominated. Thus, we get a violation of Probabilistic Admissibility.

Possibility 2. If $b$ is not a probability function, using the Joycean constraint, we get the result that there exists a probability function that weakly accuracydominates $b$, and therefore weakly accuracy-dominates $C$. Again, we get a violation of Probabilistic Admissibility.

Proposition 4. For any finite partition $\mathrm{X}$ over a set of worlds $\mathrm{W}$, let $\mathrm{C}$ and $\mathrm{C}^{*}$ be sets of probability functions in $\mathrm{B}_{\mathrm{X}}$, such that for any $\mathrm{P}$ in $\mathrm{X}, \mathrm{C}(\mathrm{P})=[\mathrm{a}, \mathrm{b}]$ and $\mathrm{C}^{*}(\mathrm{P})=$ $\{1 /|\mathrm{X}|\}$. If Strong Extensionality, Completeness, and Probabilistic Admissibility are true, then, for any legitimate imprecise global measure of accuracy $\mathrm{G}_{\mathrm{X}}{ }^{*}$ and any world $\mathrm{w}$ in $W, \mathrm{G}_{\mathrm{X}} *(\mathrm{C}, \mathrm{w})=\mathrm{G}_{\mathrm{X}} *\left(\mathrm{C}^{*}, \mathrm{w}\right)$.

Proof of Proposition 4. Strong Extensionality implies that, for any two worlds $w$ and $w^{*}, G_{X} *(C, w)=G_{X} *\left(C, w^{*}\right)$ and $G_{X} *\left(C^{*}, w\right)=G_{X} *\left(C^{*}, w^{*}\right)$. This is because $C$ and $C^{*}$ have the exact same accuracy profile in each world, since they assign $[a, b]$ and $1 /|X|$ respectively to the true propositions in every world $w$, as well as to all the false ones. Now pick one particular world $w$, and suppose that $G_{X} *(C$, $w)>\mathrm{G}_{\mathrm{X}} *\left(C^{*}, w\right)$. Then $C^{*}$ is more accurate than $C$ in every other world $w^{*}$, so $C^{*}$ weakly accuracy-dominates $C$. But this violates Probabilistic Admissibility. The same argument applies if we have $G_{X} *(C, w)<\mathrm{G}_{\mathrm{X}} *\left(C^{*}, w\right)$. Thus if we want to avoid a violation of Probabilistic Admissibility, we need to have $G_{X} *(C, w)=$ $G_{X} *\left(C^{*}, w\right)$ for every world $w$.

\section{Appendix B: Non-Numerical Measures of Accuracy}

We want to consider two distinct proposals that lay down non-numerical measures of accuracy for imprecise credal states.

The first proposal is one that Mayo-Wilson and Wheeler (2016) attribute to Joyce.

Proposal 1. For any partition $X$ over a set of worlds $W$, let $G_{X}$ be a legitimate global accuracy measure for precise credal states. Then, there exists a legitimate global accuracy measure $G_{X}$ * for imprecise credal states such that:

For any world $w$ in $W$ and any set $C$ of credence functions in $B_{X}, G_{X} *(C, w)=$ $\left\{r: \exists b\left(b \in C \& G_{X}(b, w)=r\right)\right\}$. 
On this picture, the accuracy score of an imprecise credal state is just the set of accuracy scores assigned to the individual credence functions that it involves. The problem with this proposal is that it doesn't obviously satisfy Completeness. Suppose there are two sets of probability functions $C$ and $C^{*}$ defined over a twocell partition $\{H, T\}$, such that $C(H)=C(T)=[0.2,0.8]$, and $C^{*}(H)=C^{*}(T)=$ $[0.3,0.7]$. Now, since $C^{*}$ is a subset of $C$, for any $w, G_{X} *\left(C^{*}, w\right)$ will also be a subset of $G_{X} *(C, w)$. But how do we know which set of accuracy scores is greater than the other? Since there is no natural total preorder over sets of real numbers, it is not obvious that imprecise credal states on this view have to be comparable to each other with respect to global accuracy. Thus, Completeness may indeed fail.

However, this isn't the only way we could have non-numerical measures of accuracy. Seidenfeld, Schervish, and Kadane (2012) define a local measure of inaccuracy $I$ where, for any $x$ and $y$ between 0 and 1 (inclusive), $I([x, y], 1)$ reflects the inaccuracy of assigning the set of credences $[x, y]$ to a proposition $P$ when $P$ is true, and $I([x, y], 0)$ reflects the inaccuracy of assigning $[\mathrm{x}, \mathrm{y}]$ to a proposition $P$ when $P$ is false. The inaccuracy measure $I$ is defined as follows:

$$
\begin{gathered}
I([x, y], 0)=<1+x^{2}, y^{2}> \\
I([x, y], 1)=<(1-x)^{2},(1-y)^{2}+1>
\end{gathered}
$$

Let $I([x, y], 0)<I([w, v], 0)$ iff either (i) $1+x^{2}<1+w^{2}$ or (ii) $1+x^{2}=1+w^{2}$ and $y^{2}<v^{2}$. And let $I([x, y], 1)<I([w, v], 1)$ iff either (i) $(1-x)^{2}<(1-w)^{2}$ or (ii) $(1-x)^{2}=(1-w)^{2}$ and $(1-y)^{2}+1<(1-v)^{2}+1$. This local measure of inaccuracy is lexicographic because it ranks one assignment $[w, v]$ higher than another $[x, y]$ one just in case either the first term in the score of $[w, v]$ is greater than the first term in the score of $[x, y]$ or if the first term in the scores of $[x, y]$ and $[w, v]$ are equal, but the first term in the score of $[w, v]$ is greater than the first term in the score of $[x, y]$.

On the basis of this inaccuracy measure, Seidenfeld et al (2012) proposed a global measure of inaccuracy. For any partition $X=\left\{P_{1}, P_{2}, \ldots, P_{n}\right\}$ defined over a set of worlds $\mathrm{W}, I_{X}$ is a global measure of inaccuracy, such that for any set of credence functions $C$ defined over $X$, and any world $w$ in $W, I_{X}(C, w)=<I\left(C\left(P_{l}\right), v_{w}\left(P_{1}\right)\right)$, $I\left(C\left(P_{2}\right), v_{w}\left(P_{2}\right)\right), \ldots, I\left(C\left(P_{n}\right), v_{w}\left(P_{n}\right)\right)>$. Now, we can say that $I_{X}(C, w)>I_{X}\left(C^{*}\right.$, $w)$ iff either $I\left(C\left(P_{1}\right), v_{w}\left(P_{1}\right)\right)>I\left(C^{*}\left(P_{1}\right), v_{w}\left(P_{1}\right)\right)$, or $I\left(C\left(P_{1}\right), v_{w}\left(P_{1}\right)\right)=I\left(C^{*}\left(P_{1}\right)\right.$, $\left.v_{w}\left(P_{1}\right)\right)$ and $I\left(C\left(P_{2}\right), v_{w}\left(P_{2}\right)\right)>I\left(C^{*}\left(P_{2}\right), v_{w}\left(P_{2}\right)\right)$, or $\ldots$, and so on.

In effect, Seidenfeld et al.'s proposal is this:

Proposal 2. For any partition $X$ over a set of worlds $W$, there exists a legitimate imprecise global measure of accuracy $G_{X} *$ such that:

If $C$ and $C^{*}$ are sets of credence functions defined over $\mathrm{X}$, then, for any world $w$ in $W, G_{X} *(C, w)>G_{X} *\left(C^{*}, w\right)$ iff $I_{X} *(C, w)<I_{X} *\left(C^{*}, w\right)$, and $G_{X} *(C, w)=$ $G_{X} *\left(C^{*}, w\right)$ iff $I_{X} *(C, w)=I_{X}^{*}\left(C^{*}, w\right)$.

But this proposal is incompatible with Strong Extensionality. Take a two-cell partition $X=\{H, T\}$, and let there be a set of probability functions $\mathrm{C}$ defined over $\mathrm{X}$, such that for any $P$ in $\mathrm{X}, C(P)=[0.5 ., 0.5]$. Note that $I([0.5,0.5], 0)=<1.25,0.25>$ and $I([0.5,0.5], 1)=<0.25,1.25>$. So, for any $H$-world $w, I_{X}(\mathrm{C}, w)=<<0.25,1.25>$, 
$<1.25,0.25>>$. And, for any $T$-world $w, I_{X}(C, w)=<<1.25,0.25>,<0.25,1.25>>$. Note that $C$ has the same accuracy profile in every world. But since $G_{X}(C, w)$ is not equal to $G_{X}\left(C, w^{*}\right)$ for every $w$ and $w^{*}$, Strong Extensionality fails.

\section{Appendix C: Konek's Rejection of Probabilistic Admissibility}

Lastly, we want to show that the global measure of inaccuracy Konek (forthcoming) puts forward leads to a violation of Probabilistic Admissibility. For Konek, the inaccuracy of any set of credence functions $C$ defined over a partition $X$ in a world $w$ is given by:

$$
I_{X} *(C, w)=\alpha \cdot I_{X}(a, w)+(1-\alpha) \cdot I_{X}(b, w)
$$

where $a$ is the credence function with the lowest inaccuracy score in $w$, and $b$ is the credence function with the highest score in $w$ according to the inaccuracy measure $I_{X}$ (which is just a global measure of inaccuracy based on the Brier score).

Importantly, Konek also proposes that $\alpha>1 / 2$ in order to reflect the fact that the agent values avoiding error more than seeking truth. Suppose now that $X=\{H, T\}$. Let $C_{1}$ be a set of probability functions such that for any $P$ in $X, C_{1}(P)=[0,1]$. Let $C_{2}$ be a set of probability functions such that for any $P$ in $X, C_{2}(P)=\{0.5\}$. Finally, let $C_{3}$ be a set of probability functions such that for any $P$ in $X, C_{3}(P)=$ $[0.2,0.8]$.

Now let us calculate the inaccuracy scores $I_{X} *$ assigns to these three sets of credence functions. In $C_{2}$, the most inaccurate credence function is the same as the least accurate one. So, in any $w$ in $W$,

(i) $I_{X} *\left(C_{2}, w\right)=\alpha \cdot\left(1 / 2\left((1-0.5)^{2}+(0.5-0)^{2}\right)\right)+(1-\alpha) \cdot\left(1 / 2\left((1-0.5)^{2}\right.\right.$ $\left.\left.+(0.5-0)^{2}\right)\right)=0.25$

Note two things.

(1) At any $H$-world $w$, the least inaccurate credence function in $C_{1}$ is the credence function $a_{1}$ where $a_{1}(H)=1$ and $a_{1}(T)=0$, and the most inaccurate credence function in $C_{1}$ is $b_{1}$ where $b_{1}(H)=0$ and $b_{1}(\mathrm{~T})=1$. And the least inaccurate function in $C_{3}$ is the function $a_{3}$ where $a_{3}(H)=0.8$ and $a_{3}(\mathrm{~T})=0.2$, and the most inaccurate credence function is $b_{3}$ where $b_{3}(H)=0.2$ and $b_{3}(T)=0.8$.

(2) Analogously, at any $T$-world $w^{*}$, the least inaccurate credence function in $C_{1}$ is the credence function $b_{1}$ mentioned above, and the most inaccurate credence function in $C_{1}$ is $a_{1}$ mentioned above. Similarly, the least inaccurate function in $C_{3}$ is the function $b_{3}$ mentioned above, and the most inaccurate credence function is $a_{3}$ mentioned above.

Then, for any $w$ in $W$, 
(ii) $I_{X} *\left(C_{1}, w\right)=\alpha \cdot\left(1 / 2\left((1-1)^{2}+(0-0)^{2}\right)\right)+(1-\alpha) \cdot\left(1 / 2\left((1-0)^{2}\right.\right.$

$$
\left.\left.+(0-1)^{2}\right)\right)=(1-\alpha)
$$

(iii) $I_{X} *\left(C_{3}, w\right)=\alpha \cdot\left(1 / 2\left((1-0.8)^{2}+(0.2-0)^{2}\right)\right)+(1-\alpha) \cdot\left(1 / 2\left((1-0.2)^{2}\right.\right.$

$$
\begin{aligned}
& \left.\left.+(0.8-0)^{2}\right)\right) \\
= & 0.04 \alpha+0.64-0.64 \alpha \\
= & 0.64-0.6 \alpha
\end{aligned}
$$

Now notice the following: for any $\alpha>0.75, C_{1}$ accuracy-dominates $C_{2}$, and, for any $\alpha<0.75, C_{2}$ accuracy dominates $C_{1}$. Lastly, for $\alpha=0.75, C_{3}$ accuracy-dominates both $C_{1}$ and $C_{2}$. This means that a violation of Probabilistic Admissibility is inescapable on this view.

In fact, Konek's rule will also violate Restricted Probabilistic Admissibility when $\alpha>0.5$. In order so see this, consider the accuracy scores $I_{X} *$ assigns to the doxastic state $C_{4}$ which assigns $[0.45,0.55]$ to each cell in $X$. In particular, for each world $w$

$$
\text { (iv) } \begin{aligned}
I_{S} *\left(C_{4}, w\right)= & \alpha .\left(1 / 2\left((1-0.55)^{2}+(0-0.45)^{2}\right)\right)+(1-\alpha) \cdot\left(1 / 2\left((1-0.45)^{2}\right.\right. \\
& \left.\left.+(0-0.55)^{2}\right)\right) \\
= & \alpha .0 .2025+(1-\alpha) .(0.3025) \\
= & 0.3025-\alpha .0 .1025
\end{aligned}
$$

Note that for $\alpha>0.53, I_{X} *\left(C_{4}, w\right)=0.248175<0.25=I_{X} *\left(C_{2}, w\right)$ for each world $w$. In other words, $C_{4}$ accuracy-dominates the precise probabilistic belief state $C_{2}$. Note that we need Restricted Probabilistic Admissibility to be true in order for Joyce's accuracy-dominance argument for Probabilism to go through. Hence, if we want to preserve that argument, we should reject Konek's inaccuracy measure for imprecise credal states. ${ }^{32}$

\section{Notes}

${ }^{1}$ See, e.g., Howson and Urbach (2006), Bovens and Hartmann (2003), and Talbott (2016).

${ }^{2}$ See, e.g., Jeffrey (1983), and van Fraassen (1990). Also, see Pfeifer and Kleiter (2007), Wallsten and Budescu (1995), Smithson and Pushkarskaya (forthcoming) for applications of imprecise probabilities to psychology, linguistic processing and neurological responses to ambiguity and conflict.

${ }^{3}$ See e.g. Levi (1974,1980, 1985), Walley (1991), Joyce (2005, 2010), Weatherson (ms.), Sturgeon (2008), Hájek \& Smithson (2012), and Moss (2014). For a survey of some of the different arguments for having imprecise credences, see Bradley (2016).

${ }^{4}$ A case like MYSTERY COIN is discussed by Joyce (2010, p. 283); this particular case is discussed by Schoenfield (2015) and Carr (2015).

${ }^{5}$ In other words, if, given your evidence, it is possible that the objective chance of the coin landing heads is $3 / 4$, then $3 / 4$ should be in the set of credences that you assign to the proposition that the coin will land heads.

${ }^{6}$ Permission for Imprecision follows from Requirement of Imprecision when we assume that any credal state that is rationally required must also be rationally permissible. Weak Requirement of Imprecision follows when we assume that there are some sets of propositions $X$, with respect to which an agent can have the relevant kind of non-specific evidence.

${ }^{7}$ See, e.g., Joyce (2009) and Pettigrew (2016). 
${ }^{8}$ For accuracy-based arguments for Probabilism, see Joyce (1998, 2009), Leitgeb and Pettigrew (2010), and Pettigrew (2016). For arguments for Conditionalization, see Greaves and Wallace (2006), Easwaran (2013), and Briggs and Pettigrew (forthcoming).

${ }^{9}$ A credence function defined over a partition $X$ on $W$ will assign a real number between 0 and 1 (inclusive) to every cell of $W$. A probability function defined over a partition $X$ is a credence function defined over $X$ such that the values it assigns to the cells in $X$ sum to 1 .

${ }^{10}$ Joyce (2009) defends a restricted version of Probabilistic Admissibility for precise probability functions under the label of Coherent Admissibility.

${ }^{11}$ One assumption of this argument is that if a credal state is weakly accuracy-dominated, then it cannot be rationally permissible for an agent to adopt it. Some writers such as Pettigrew (2013) deny this assumption: they argue that we aren't rationally required to avoid dominated options, but rather options that are dominated by non-dominated options. This is what Pettigrew calls Undominated Dominance. Even though our present argument for Probabilistic Admissibility doesn't straightforwardly succeed with Undominated Dominance, we can still construct a slightly more fleshed out argument that is compatible with this principle. It goes like this.

Suppose a probabilistic credal state represented by a set of probability functions $C$ is weakly accuracydominated by another credal state represented by a set of credence functions $C^{*}$. Also, suppose the state represented by $C$ is rationally permissible, because the only evidence that the agent has regarding the relevant partition is that the objective chance function for the relevant partition could be any of the probability functions belonging to $C$. Note that the following principle seems to be true: if a credal state $C$ is rationally permissible, and $C^{*}$ weakly accuracy-dominates $C$, then $C^{*}$ is rationally permissible. Now, either the state represented by $C^{*}$ is probabilistic or it's not. If it is non-probabilistic, then the principle in question yields the conclusion that the credal state represented by $C^{*}$ is rationally permissible in some situation despite being non-probabilistic. This is a bad result. Moreover, even if the state represented by $C^{*}$ is probabilistic, then there is no reason why $C^{*}$ must be rationally permissible. For there might be some objective chance distribution $p$ in $C^{*}$ which the agent's evidence rules out, or there might be some chance distribution $p$ which $C^{*}$ doesn't contain but the agent's evidence doesn't rule out. In either case, intuitively, the credal state given by $C^{*}$ will be rationally impermissible for the agent to adopt. But note that one of these possibilities has to be true if $C^{*}$ is distinct from $C$. Therefore, if $C$ represents a probabilistic credal state, it cannot be weakly accuracy-dominated by another state represented by $C^{*}$ (whether or not that latter state is itself dominated).

${ }^{12}$ Schoenfield doesn't use World-Invariance in her proof, because she doesn't make the distinction between the set of worlds $W$ and the partition $X$ over that set. As a result, she does not evaluate the accuracy of credence functions or sets of credence functions at worlds in $W$, but rather at propositions within the relevant partition $X$. Since we do not do this, we need World-Invariance in order to reconstruct her proof of Proposition 1.

${ }^{13}$ Schoenfield $(2015$, p. 5). This constraint is also subject to an objection similar to the Bronfman objection to the Non-Dominance Principle that we discuss later. See footnote 20 .

14 The proof of Proposition 1, along with all other proofs, is given in the appendix.

15 Joyce (2009) explicitly defends Strict Immodesty as a desirable property of global measures of accuracy for precise credal states. See also Oddie (1997), Greaves and Wallace (2006), and Gibbard (2008).

16 This version of Truth-Directedness is also accepted by other authors, such as Mayo-Wilson and Wheeler (2016) .

${ }^{17}$ Note that Local Admissibility could be strengthened by making the relevant kind of dominance weak dominance but we only need this weaker constraint.

18 The reasoning here importantly presupposes a principle similar to the constraint called Local-

Global Connection that we discuss below. According to this principle, for any legitimate imprecise local measure of accuracy $L^{*}$, there exists a legitimate imprecise global measure of accuracy $G_{X}{ }^{*}$, such that for any two sets $C$ and $C^{*}$ of credence functions defined over X, and any world $w$ in $W$, if $L^{*}\left(C(P), v_{w}(P)\right)$ $\geq L^{*}\left(C^{*}(P), v_{w}(P)\right)$ for every proposition $P$ in $X$ and $L^{*}\left(C\left(P^{*}\right), v_{w}\left(P^{*}\right)\right)>L^{*}\left(C^{*}\left(P^{*}\right), v_{w}\left(P^{*}\right)\right)$ for some proposition $P^{*}$ in $X$, then $G_{X} *(C, w)>G_{X} *\left(C^{*}, w\right)$. This principle is motivated by the same considerations that motivate Local-Global Connection, and we think that everything that we say in defense of 
Local-Global Connection in this section and $\$ 7$ will equally apply to this principle. So, anyone who accepts Local-Global Connection should also be fine with this variant.

${ }^{19}$ For example, Pettigrew (2016) defends a principle called Additivity, and Joyce (2009) endorses a principle called Separability, both of which entail (i). However, an advantage of the Local-Global Connection over Additivity and Separability is that the latter two constraints entail that accuracy is not a dependent good, i.e., the manner in which a particular credence in a particular proposition contributes to the accuracy of the agent's total doxastic state doesn't depend on what other credences she has in other propositions. But Local-Global Connection doesn't require us to accept anything as strong as that.

${ }^{20}$ This principle is in fact controversial. On the one hand, it is subject to what has come to be known as the Bronfman objection: Bronfman (ms.) argues that a doxastic state needn't be rationally impermissible to adopt merely because it is dominated by some other doxastic state on every legitimate accuracy measure; for there might not be any one doxastic state which dominates it on every legitimate accuracy measure. For a reply, see Pettigrew (2016). On the other hand, it is also attacked by Pettigrew (2013) who defends Undominated Dominance instead. See, for discussion, footnote 11. Note that our argument here will go through even with Undominated Dominance; for we show in our proof of Proposition 3 that some imprecise probabilistic credal states are accuracy-dominated by probabilistic precise credal states. Since probabilistic credal states are not dominated by other credal states (by Probabilistic Admissibility), the relevant imprecise credal states will be ruled out by Undominated Dominance.

${ }^{21}$ Also, see Leitgeb and Pettigrew (2010), and Pettigrew (2016).

${ }^{22}$ Proposition 3 also undermines plausible views that recognize epistemic values besides accuracy, but nevertheless want to admit that accuracy is an important goal of credences. One example of such a view is the one developed by Mayo-Wilson and Wheeler (2016) who hold that while considerations of accuracy don't favor imprecise credences in such situations as MYSTERY COIN, the fact that they fit better with the unspecific nature of the agent's evidence is a reason to adopt these credences in cases when precise credences and imprecise credences fare equally well with respect to accuracy. Proposition 3 undermines their argument because it shows that at least some seemingly rational imprecise credal states are accuracy-dominated by precise ones, and therefore don't fare equally well as those precise states with respect to accuracy.

${ }^{23}$ We discuss two proposals of this kind in Appendix B.

${ }^{24}$ A multiset is a collection that, unlike a set, allows multiple instances of its elements. For example, $\{a, a, b\}$ and $\{a, b\}$ are different multisets. However, order does not matter, so $\{a, a, b\}$ and $\{a, b, a\}$ are the same multiset.

${ }^{25}$ Pettigrew (2016) and Schoenfield (2015) accept Strong Extensionality.

${ }^{26}$ Our statement of this constraint involves some abuse of notation. Presumably, the greater than relation denoted by ' $>$ ' only holds between numerical values. However, we are assuming that it also holds between non-numerical values. However, this use of notation is harmless, since we could restate everything we have said using a different greater than relation denoted by ' $\succ$ '.

${ }^{27}$ Note that someone who wants to allow for the rationality of a credal state that assigns $[0,1]$ to each cell of a partition may face other problems, e.g., those discussed by Rinard (2013). However, the problem we are raising doesn't depend on the fact that the agent assigns $[0,1]$ to each cell of a four-cell partition; the problem could arise even if the assignment were [0.1., 0.7].

${ }^{28}$ An additional problem might be that Proposition 4 (along with the Permission Principle, Completeness, Strong Extensionality, and Probabilistic Admissibility) implies that the Principal Principle is false (see also Schoenfield (2015)). In order to see why this is so, consider two different probabilistic credal states, represented by the sets of credence functions $C_{1}$ and $C_{2}$ defined over an $n$-cell partition $X$, such that for any $P$ in $X, C_{1}(P)=[0,1]$ and $C_{2}(P)=\{1 / n\}$. Now, suppose an agent has conclusive evidence that the objective chance of any $P$ in $X$ is $1 / n$. So, she is required by the Principal Principle to adopt the credal state represented by $C_{2}$ in this scenario (provided that she doesn't possess any inadmissible information). But, given Proposition 4, Completeness, Strong Extensionality, and Probabilistic Admissibility, both these credal states are equally accurate in every world. Therefore, Proposition 4, Completeness, Strong Extensionality, and Probabilistic Admissibility (together with the Permission Principle) conflict with the Principal Principle.

However, we suspect that this problem may not ultimately be unsurmountable. Here is a possible strategy. If the agent adopts an imprecise credal state in a scenario where she has conclusive evidence 
about what the chance distribution over the relevant partition is, a number of credence functions in the representor (i.e., the set of credence functions that represents her credal state) will violate the Principal Principle, so the overall imprecise state will also be irrational. (In the next section, we give a similar argument for ruling out the rationality of non-probabilistic imprecise credal states by appealing to a principle called Rational Admissibility. We argue that since the set of credence functions that represents a non-probabilistic imprecise credal state contains probabilistically incoherent credence functions, and probabilistically incoherent credence functions are rationally impermissible to adopt for accuracy-based reasons, such non-probabilistic imprecise credal states are rationally impermissible to adopt even though they match other probabilistic credal states with respect to global accuracy.) Assuming that there are accuracy-based arguments for the Principal Principle (e.g., the ones discussed by Pettigrew $(2012,2016)$ ), we could indeed show that there is some accuracy-based disadvantage to adopting an imprecise credal state which is represented by a set of credence functions that are not all compatible with the Principal Principle.

${ }^{29}$ In this context, rejecting Probabilistic Admissibility could be counter-productive. Consider two different probabilistic credal states, represented by the sets of credence functions $C_{1}$ and $C_{2}$ defined over an $n$-cell partition $X$, such that for any $P$ in $X, C_{1}(P)=[a, b]$ and $C_{2}(P)=\{1 / n\}$. Proposition 4 (along with the other constraints) entails that these two credal states have the same global accuracy score in every world. If the defender of imprecise credence rejects Probabilistic Admissibility in this context, she would have to allow one of the credal states to strongly accuracy-dominate the other. If the imprecise credal state accuracy-dominates the other state, then, by the Non-Dominance Principle, she will now be committed to claim that from an accuracy-centered standpoint, it is never rationally permissible for an agent to adopt a probabilistically coherent credal state that assigns $1 / n$ to each cell of an n-cell partition. But there are cases where the Principal Principle will require such a credal state, so that seems bad. If the precise credal state accuracy-dominates the other state, then, by the Non-Dominance Principle, the defender of imprecise credences will now be committed to claim that from an accuracy-centered standpoint, it is never rationally permissible for an agent to adopt a probabilistically coherent credal state that assigns an interval $[a, b]$ to all the cells of an $n$-cell partition. This will be inconsistent with the natural precisification of Permission for Imprecision that we discussed in $\$ 4.2$, thus leaving some of the problems for the defender of imprecise credences intact.

${ }^{30}$ Here is why. Each of these two credal states has the same accuracy profile in every possible world and therefore, by Strong Extensionality, they have the same global accuracy score in every world. Hence, if the defender of imprecise credences wants to avoid violations of Probabilistic Admissibility, she must say either that they match each other with respect to global accuracy in every world or that they are incomparable everywhere. Since the first option is bad for reasons discussed earlier, the defender of imprecise credences must say that they are incomparable with respect to global accuracy in every world.

${ }^{31}$ Recall that the motivation for Local Admissibility also appeals to similar considerations. So, if we reject Local-Global Connection, we may also have to reject Local Admissibility.

${ }^{32}$ Acknowledgements: The authors would like to thank Malcolm Forster, Daniel Kokotajlo, Matthew Kotzen, Lu Teng, audiences at the NYU Shanghai Epistemology Reading Group, and especially an anonymous referee, for helpful comments on earlier versions of this paper.

\section{References}

Bovens, Luc, and Stephan Hartmann. (2003). Bayesian Epistemology. Oxford: Oxford University Press. Bradley, Seamus. (2016). Imprecise Probabilities. In Edward N. Zalta (ed.), The Stanford Encyclopedia of Philosophy (Winter 2016 Edition). https://plato.stanford.edu/archives/win2016/ entries/imprecise-probabilities

Briggs, R.A., and Pettigrew, Richard. (forthcoming). An Accuracy-Dominance Argument for Conditionalization. Nous.

Bronfman, A. (ms.). A Gap in Joyce's Argument for Probabilism.

Carr, Jennifer. (2015). Chancy accuracy and imprecise credence. Philosophical Perspectives 29 (1):67-81.

deFinetti, Bruno. (1937). La Prévision: Ses Lois Logiques, Ses Sources Subjectives. Annales de l'Institut Henri Poincaré 17:1-68. 
Easwaran, Kenny. (2013). Expected Accuracy Supports Conditionalization - and Conglomerability and Reflection. Philosophy of Science 80 (1):119-142.

Greaves, Hilary \& Wallace, David. (2006). Justifying conditionalization: Conditionalization maximizes expected epistemic utility. Mind 115 (459):607-632.

Gibbard, Alan. (2008). Rational Credence and the Value of Truth. In Tamar Gendler and John Hawthorne, (eds.), Oxford Studies in Epistemology, Volume 2. Oxford: Oxford University Press.

Hájek, Alan, and Michael Smithson. (2012). Rationality and Indeterminate Probabilities. Synthese, 187: 33-48.

Howson, Colin, and Peter Urbach. (2006). Scientific Reasoning: the Bayesian Approach. Chicago: Open Court.

Jeffrey, Richard C. (1983). Bayesianism with a Human Face. In John Earman (ed). Testing Scientific Theories. Minneapolis: University of Minnesota Press.

Joyce, James M. (1998). A Nonpragmatic Vindication of Probabilism. Philosophy of science, 65(4), $575-603$.

. (2005). How Probabilities Reflect Evidence. Philosophical Perspectives 19 (1):153-178.

(2009). Accuracy and Coherence: Prospects for an Alethic Epistemology of Partial Belief. In Franz Huber \& Christoph Schmidt-Petri (eds.), Degrees of Belief. Synthese. pp. 263297.

(2010). A Defense of Imprecise Credences in Inference and Decision making. Philosophical Perspectives 24 (1):281-323.

Konek, Jason. (forthcoming). Epistemic Conservativity and Imprecise Credence. Philosophy and Phenomenological Research.

Leitgeb, Hannes \& Pettigrew, Richard. (2010). An Objective Justification of Bayesianism II:Consequences of Minimizing Inaccuracy. Philosophy of Science 77 (2):236-272.

Levi, Isaac. (1974). On Indeterminate Probabilities. Journal of Philosophy, 71: 391-418.

(1980). The Enterprise of Knowledge: An Essay on Knowledge, Credal Probability, and Chance. MIT Press.

(1985). Imprecision and Indeterminacy in Probability Judgment. Philosophy of Science, 52: 390-409.

Mayo-Wilson, Conor \& Wheeler, Gregory. (2016). Scoring Imprecise Credences: A Mildly Immodest Proposal. Philosophy and Phenomenological Research 92 (1):55-78.

Moss, Sarah. (2014). Credal Dilemmas. Noûs 48 (3):665-683.

Oddie, Graham. (1997). Conditionalization, Cogency, and Cognitive value. British Journal for the Philosophy of Science 48 (4):533-541.

Pettigrew, Richard. (2012). Accuracy, Chance, and the Principal Principle. Philosophical Review 121 (2):241-275.

(2013). Accuracy and Evidence. Dialectica 67 (4):579-596.

(2016). Accuracy and the Laws of Credence. Oxford: Oxford University Press.

Pfeifer, Niki, and Gernot D. Kleiter. (2007). Human Reasoning with Imprecise Probabilities: Modus Ponens and Denying the Antecedent. Proceedings of the 5th International Symposium on Imprecise Probability: Theory and Application: 347-356.

Rinard, Susanna. (2013). Against Radical Credal Imprecision. Thought: A Journal of Philosophy 2 (1):157-165.

Schoenfield, Miriam. (2015). The Accuracy and Rationality of Imprecise Credences. Noûs 50 (4).

Seidenfeld, T., Schervish, M. J., \& Kadane, J. B. (2012). Forecasting with Imprecise Probabilities. International Journal of Approximate Reasoning, 53(8), 1248-1261.

Smithson, Michael, and Helen Pushkarskaya. (forthcoming). Ignorance and the Brain: Are there Distinct Kinds of Unknowns? In Matthias Gross and Linsey McGoey (eds), Routledge International Handbook of Ignorance Studies. London and New York: Routledge.

Sturgeon, Scott. (2008). Reason and the Grain of Belief. Noûs 42 (1):139-165.

Talbott, William. (2016). Bayesian Epistemology. In Edward N. Zalta (ed.), The Stanford Encyclopedia of Philosophy (Winter 2016 Edition). https://plato.stanford.edu/archives/win2016/ entries/epistemology-bayesian 
van Fraassen, Bas. (1990). Figures in a Probability Landscape. In J. Dunn \& A. Gupta (eds.), Truth or Consequences. Dodrecht: Kluwer.

Walley, Peter. (1991). Statistical Reasoning with Imprecise Probabilities, Monographs on Statistics and Applied Probability, Vol. 42. London: Chapman and Hall.

Wallsten, Thomas, and David V. Budescu. (1995). A Review of Human Linguistic Probability Processing:

General Principles and Empirical Evidence. The Knowledge Engineering Review, 10: 43-62.

Weatherson, Brian. (ms.) Decision Making with Imprecise Probabilities. Unpublished manuscript. 